\title{
Rheology of Polymer Brushes: A Brownian Dynamics Study
}

\author{
Patrick S. Doyle, Eric S. G. Shaqfeh,* and Alice P. Gast \\ Department of Chemical Engineering, Stanford University, Stanford, California 94305 \\ Received J une 9, 1997; Revised Manuscript Received May 1, 1998
}

\begin{abstract}
We present results of Brownian dynamics simulations of polymer brushes under steady and oscillatory shear. The brush is sheared by a bare surface and the resulting solvent velocity and polymer dynamics are solved self-consistently. Under steady shear the deformation of the brush proceeds in two steps: chains tilt in the flow direction followed by a physical thinning of the brush. The brusheffective viscosity increases upon compression to near $60 \%$ and decreases thereafter. We develop a scaling based on the Brinkman equation to explain the unusual trends in the viscosity. Upon introducing oscillatory shear flow in the brush, we observe large increases in the normal stress and bead density near the upward surface. Shear-induced collisions of beads in the brush increase the osmotic pressure and thus give rise to these normal forces. The strain amplitude determines the dynamics during oscillatory flow, and we develop scalings for the range of strain amplitude over which the normal stress increases occur. The simulation results for a single grafted layer are compared to the experiments performed by Klein et al. for the shearing of two grafted layers.
\end{abstract}

\section{Introduction}

Polymers which are constrained by one end to a surface will adopt different configurations than when in free solution. At large coverages the polymers will tend to stretch away from the surface to form a polymer brush. Tethered polymers have been utilized extensively for stabilizing both naturally occurring colloids (such as Casein micelles in milk ${ }^{3}$ ) and synthetic colloids (such as latex particles in paints ${ }^{4}$ ). Recent experimental studies have shown that the rheology of the colloids can be greatly influenced by the tethered chains through enhanced slip at the fluid-particle interface ${ }^{5}$ or possibly changing the suspension microstructure ${ }^{6}$ and lubrication properties ${ }^{7}$ at large shear rates.

More recently, the rheology of the polymer brushes themselves have become of interest. Klein et al..$^{1,2}$ and Pelletier et al. ${ }^{8}$ have measured the normal and shear forces of sheared polymer brushes in good solvents using the surface force apparatus, SFA. K lein et al..$^{1,2}$ studied the shear of a brush-brush system while the shear experiments of Pelletier et al. ${ }^{8}$ where performed on a brush-mica system. Klein et al. ${ }^{1}$ demonstrated that substantial normal force increases can be induced under the application of oscillatory flow at large shear rates and frequencies (i.e., in the nonlinear deformation regime) for both compressed and uncompressed brushes. At small shear rates and frequencies (i.e., in the linear deformation regime) Pelletier et al. ${ }^{8}$ found that the normal forces for a compressed brushed (compressed to $50-90 \%$ of the equilibrium thickness) do not change from the equilibrium value. Furthermore, they found that the storage modulus is larger than the loss modulus (at a fixed frequency of $10 \mathrm{~Hz}$ ) and both increase upon compression of the brush. Pelletier et al. ${ }^{8}$ were unable to detect any tangential (shear) forces in their experiments. During slow steady shearing, Klein et al. ${ }^{2}$ found that the normal forces did not change from their equilibrium values and the shear stress was small (bel ow the detection of the apparatus) until the brushes were compressed to $10-15 \%$ of their equilibrium thickness at which point they increased rapidly.
The previous SFA experiments are complementary and provide the following experimental overview for the rheology of a polymer brush in a good solvent: in the linear deformation regime a compressed polymer brush (in either a brush-brush or brush-mica configuration) does not change thickness and shear forces are small (unmeasurable) for moderate compressions, shear forces become significant (though smaller than normal forces) when a brush-brush system is compressed beyond 10$15 \%$ of the equilibrium thickness, oscillation of a compressed and uncompressed polymer brush (in a brush-brush system) in the nonlinear deformation regime gives rise to increases in the normal forces.

Though we will only simulate good solvent conditions, it is interesting to mention the systematic work that has been performed by Granick and co-workers ${ }^{9-11}$ on the linear viscoelastic response of polymer brushes in near $\Theta$ solvent conditions using the SFA. Shear forces are much smaller than the normal forces until the brushes (in a brush-brush system) are compressed (approximately $30-50 \%$ of the equilibrium thickness depending on the chain lengths and brush density). This is in qualitative agreement with the experiments for a brush in a good solvent, but differs from the good solvent case in that less compression is needed to see this increase. Granick et al. ${ }^{11}$ also compared the rheology of a brush-mica system to that of a brush-brush system. The systems are similar in that shear forces are much smaller than the normal forces; however, a brush-mica system must be compressed substantially more (to approximately $10 \%$ of the equilibrium thickness) before a substantial rise in the shear forces is detected. Furthermore, at compressions less than 35\% the shear elastic modulus divided by the normal pressure is smaller in the brush-mica system than in the brush-brush system. This difference is most probably due to the interpenetration of the brushes in the brushbrush system. ${ }^{11}$

Many studies ${ }^{12-16}$ have proposed explanations for the normal force increases observed by Klein et al. $^{1}{ }^{1} \mathrm{~K}$ umaran $^{12}$ proposed that the asymmetric pair distribution of monomers produced by the flow is unstable to hydrodynamic interactions and the brush increases in thick- 
ness (up to a $33 \%$ increase). Rabin and Alexander ${ }^{13}$ considered a tangential force applied to the end of an Alexander de Gennes step-profile brush. They calculated that the thickness of an uncompressed brush is independent of shear rate while a compressed brush will increase in thickness at large shear rates. Barrat ${ }^{14}$ showed that the theory of Rabin and Alexander ${ }^{13}$ can predict brush thickening (up to a $25 \%$ increase) due to increased excluded volume interactions as the chains are stretched. The concept proposed by Barrat was refined by Harden and Cates ${ }^{15}$ who calculated the polymer deformation and velocity profile via the brush in a self-consistent procedure. Harden and Cates ${ }^{15}$ al so predict swelling of the brush (up to a $25 \%$ increase) during steady shear. The previous theory of Harden and Cates was recently modified by Aubouy, Harden, and Cates $^{17}$ to include two classes of chains: one set is exposed to the flow and the other set is shielded within the brush. This new model predicts a larger brush swelling (up to a $50 \%$ increase) which occurs at a smaller shear rate than that in the original model. Recent simulations by Doyle et al. ${ }^{16}$ demonstrated that oscillation of a polymer brush leads to shear-induced collisions within the brush, giving rise to shear thickening. In this paper we will extend the study of Doyle et al. ${ }^{16}$ to provide a more comprehensive description of polymer brush rheology and introduce scalings to describe several of the observed behaviors.

The study of hydrodynamic properties of polymer brushes has its origins in earlier investigations of adsorbed polymer layers (polymers which are attached at multiple points to a surface to form trains, loops, and tails). These studies ${ }^{18-20}$ have al most exclusively proceeded by treating the layer as a porous medium and invoking the Brinkman equation:21

$$
\frac{\partial^{2} u_{i}}{\partial x_{j} \partial x_{j}}-\frac{\partial P}{\partial x_{i}}-\mu \kappa^{2} u_{i}=0
$$

where $\mu$ is the solvent viscosity, $\mathrm{P}$ is the pressure, $\mathrm{u}_{\mathrm{i}}$ is velocity, and $\kappa$ is the pore size. The pore size is the characteristic distance over which the velocity decays. For the polymer brush $\kappa$ is assumed to be a function of the polymer density. The term $\mu \kappa^{2} u_{i}$ in eq 1 accounts for the hydrodynamic resistance of the adsorbed chains. We note that more precise descriptions of the hydrodynamics of adsorbed layers do exist (see, for example, the nonlocal hydrodynamic theory of Wu and $\mathrm{Cates}^{22}$ ) but have not been widely utilized due to their complexities. Most studies ${ }^{18,19}$ have assumed the flow does not alter the polymer concentration profile or have allowed the concentration profile to change on the basis of a phenomenological model. 20 More recently, Brownian dynamics simulations ${ }^{23}$ of dilute tethered chains (representing the "tails" portion of the adsorbed layer) have calculated the solvent velocity and polymer concentration profile self-consistently.

Fewer theoretical studies have been performed of polymer brush hydrodynamics. Fredrickson and Pincus $^{24}$ considered a lubrication analysis of the Brinkman equation for compressed polymer layers which was in fair agreement with surface force experiments of Klein et al. ${ }^{25}$ Milner ${ }^{26}$ showed that there is greater hydrodynamic penetration into the SCF parabolic brush 27,28 than the Alexander de Gennes step brush.29,30 These two studies did not take into account the deformation of the polymer brush due to flow. Harden and Cates ${ }^{15}$ and Aubouy, Harden, and Cates ${ }^{17}$ calculated the deformation of the brush and the velocity field self-consistently. Kumaran ${ }^{12}$ examined the effect of hydrodynamic interactions of monomers in the brush when the pair potential is asymmetric due to shear deformation. The theories of Harden et al., ${ }^{15}$ Aubouy et al., ${ }^{17}$ and Kuma$\operatorname{ran}^{12}$ all predict shear-induced swelling of polymer brushes.

Stochastic simulations are a powerful tool to study nonequilibrium polymer dynamics. Simulations of polymer brushes under steady shear were first performed by Lai and Binder ${ }^{31}$ using a lattice bond fluctuation Monte Carlo method where the flow is incorporated via an enhanced jump rate in the flow direction. They solved the Brinkman equation ${ }^{21}$ to determine the flow and density profile in the brush self-consistently. They found that the brush thickness decreased slightly $(\sim 2 \%)$ for the largest shear rates. Recently, Lai and Lai 32 modified the algorithm of Lai and Binder to have the blob size input into the Brinkman equation depend on shear force and not the volume fraction of monomers. They present scalings for the stretch of the chain in the flow direction and report that no significant change in the brush height was observed. Another Monte Carlo simulation of polymer brushes was recently performed by Miao, Guo, and Zuckerman ${ }^{33}$ using an off-lattice algorithm and a self-consistent calculation of the flow profile. They studied the tilting and stretching of the chains and found favorable agreement with the theory of Rabin and Alexander. ${ }^{13}$ The brush thickness and bead density profile were unaffected by the shear flow over the range of shear rates they simulated.

Molecular dynamics simulations of sheared polymer brushes were performed by Peters and Tildesley ${ }^{34}$ which included explicit solvent molecules. They studied an uncompressed brush system consisting of chains of length $\mathrm{N}=20$ at a high surface coverage of $33 \%$. The radius of gyration perpendicular to the walls decreased with increasing shear rate while the component parallel to the walls increased. Peters and Tildesley ${ }^{35}$ recently extended their study of the same system $(N=20)$ in which some chains were al lowed to detach from the wall to study the shear of the tethered chain with free polymers. Shear stresses were largest at intermediate converges which was attributed to entanglements between the brushes and free chains.

Grest has performed a series of molecular dynamics simulations of sheared polymer brushes with a continuum solvent ${ }^{36}$ and with explicit solvent molecules (dimers). ${ }^{37}$ Qual itatively, the results did not differ. He studied both compressed and uncompressed brushes for chains of length $\mathrm{N}=100$ where $\mathrm{N}$ is the number of beads in a chain. At high shear rates the brushes stretch in the flow direction and disentangle. No brush thickening was ever witnessed. The normal and shear forces were in qualitative agreement with the steady shear experiments of Klein et al.2

Recently, Neelov et al. ${ }^{38}$ have performed molecular dynamics simulations of a single uncompressed polymer brush subject to a tangential uniform flow field. The brush is assumed to be freely draining. For small flow rates they find a $3 \%$ increase in the perpendicular components to the radius of gyration and end-to-end distance of the chains for a dilute brush of length $\mathrm{N}=$ 50. At larger flow rates, areal fractions, and chain lengths the dimensions of the brush in the direction perpendicular to the surface decreased. 
The previous simulations, except the study of Neelov et al., 38 found constant or decreased brush heights during steady shearing which agrees with the theory of Rabin and Alexander; ${ }^{13}$ however, this feature directly disagrees with the more recent theories of Barrat,14 Kumaran, ${ }^{12}$ Harden and Cates, ${ }^{17}$ and Aubouy et al. ${ }^{17}$ Furthermore, the rheological properties of the layers have only been addressed by a few authors ${ }^{16,34-37}$ and demand further study.

In this paper, we present results of Brownian dynamics simulations of a polymer brush under steady and oscillatory shear. We consider the case of a surface containing tethered chains which is sheared by a bare surface. We devel op a simulation method to calculate the solvent velocity in the brush and the polymer dynamics consistently. The conformation and rheology of brushes under steady and oscillatory shear are compared. We present scalings for the critical frequencies and shear rates where shear thickening during oscillation is witnessed. We compare our simulation results to the experiments of Klein et al. ${ }^{1,2}$

\section{Simulation Method}

The simulated system consists of polymer chains tethered to a solid flat surface which are sheared via motion of a top solid surface. The simulation is a modification of the our single "free" chain, Brownian dynamics algorithm described in a previous publication. ${ }^{39}$ The brush is composed of $\mathrm{N}_{\mathrm{c}}$ chains containing $\mathrm{N}$ beads each. The polymers are model ed as beadrod chains, where the beads act as sources of friction and the rods serve as constraints to hold successive beads at a constant relative distance. Physically, the rod length scal e corresponds to a Kuhn step in a polymer molecule and is a measure of the chain rigidity.

We employ index notation throughout our discussion and Greek superscripts will refer to bead numbers. A stochastic differential equation used to compute chain trajectories can be derived by considering the relevant forces on the chain: hydrodynamic $\left(F_{i}^{\mathrm{h}, \nu}\right)$, Brownian $\left(\mathrm{F}_{\mathrm{i}}^{\mathrm{br}, \nu}\right)$, constraint $\left(\mathrm{F}_{\mathrm{i}}^{\mathrm{c}, \nu}\right)$, excluded volume $\left(F_{i}^{\mathrm{ev}, v}\right)$, wall $\left(\mathrm{F}_{i}^{\text {wall, }, v}\right)$, and tethering $\left(\mathrm{F}_{t}^{\mathrm{t}, v}\right)$. Neglecting inertia, the forces on a bead are summed and set to zero. The solvent is treated as a continuum.

The hydrodynamic force on a bead is assumed to be linear in the slip vel ocity between the bead and the solvent velocity at the bead center. Thus,

$$
\mathrm{F}_{\mathrm{i}}^{\mathrm{h}, v}=-\zeta\left(\frac{\mathrm{dr} r_{\mathrm{i}}^{v}}{\mathrm{dt}}-\left\langle\mathrm{v}_{\mathrm{i}}\left(\mathrm{r}_{\mathrm{i}}^{v}\right)\right\rangle\right)
$$

where $\xi$ is the drag on a bead, $r_{i}^{r}$ is the location of the center of the bead $v$, and $\left\langle\mathrm{v}_{\mathrm{i}}\left(\mathrm{r}_{\mathrm{i}}^{v}\right)\right\rangle$ is the ensemble-averaged solvent velocity at position $\mathrm{r}_{\mathrm{i}}^{\nu}$. We set $\xi=3 \pi \mu$ a where $\mu$ is the sol vent viscosity and $a$ is the bead diameter. We will return to a discussion of $\left\langle v_{i}\left(r_{i}^{v}\right)\right\rangle$ in due course.

We assume that during each time step a bead experiences numerous coll isions with the solvent molecules. These Brownian forces are approximated as a $\delta$-correlated, white noise process. ${ }^{40}$ A discrete form for the Brownian forces during an individual time step beginning at time t and ending at time $t$ $+\delta \mathrm{t}$ is

$$
\begin{gathered}
\left\langle\mathrm{F}_{\mathrm{i}}^{\mathrm{br}, v}\left(\mathrm{t}^{*}\right)\right\rangle=0 \\
\left\langle\mathrm{~F}_{\mathrm{i}}^{\mathrm{br}, \nu}\left(\mathrm{t}^{*}\right) \mathrm{F}_{\mathrm{j}}^{\mathrm{br}, \mu}\left(\mathrm{t}^{*}\right)\right\rangle=\frac{2 \mathrm{kT} \zeta \delta_{\nu \mu} \delta_{\mathrm{ij}}}{\delta \mathrm{t}}
\end{gathered}
$$

where $t^{*}$ is equal to $t+\delta \mathrm{t} / 2$ which corresponds to a Stratonavich interpretation of the stochastic term. ${ }^{40}$ The $2 \mathrm{kT} \xi$ term results from satisfying the fluctuation dissipation theorem. ${ }^{41}$
The constraint forces are calculated using the method of Lagrangian multipliers employed by Liu. ${ }^{42}$ The constraint force on bead $v$ is

$$
\mathrm{F}_{\mathrm{i}}^{\mathrm{c}, v}=\mathrm{T}^{v} \mathrm{u}_{\mathrm{i}}^{\nu}-\mathrm{T}^{\nu-1} \mathrm{u}_{\mathrm{i}}^{\nu-1}
$$

where $T^{v}$ are the $\mathrm{N}-1$ undetermined Lagrangian multipliers, $u_{i}^{v}=\left(r_{i}^{v+1}-r_{i}^{v}\right) / a$, and the rod length is equal to the bead diameter. The Lagrangian constraints are chosen to satisfy the constraint of constant rod length at the end of a time step.

We model the excluded volume force using the repulsive part of a Lennard-J ones potential. ${ }^{43}$ The dimensionless excluded volume force on bead $v$ due to bead $\iota$ takes the form

$$
\begin{aligned}
& F_{i}^{e v, v}=24 \epsilon\left(r_{i}^{v}-r_{i}^{l}\right)\left[2\left(\frac{d^{\prime}}{\left|r_{i}^{v}-r_{i}^{l}\right|}\right)^{14}-\left(\frac{d^{\prime}}{\mid r_{i}^{v}-r_{i}^{l}}\right)^{8}\right] \text { for } \\
&\left|r_{i}^{v}-r_{i}^{l}\right| \leq 2^{1 / 6} a
\end{aligned}
$$

where $\epsilon$ is the depth of the Lennard-J ones potential well and $d^{\prime}$ is the coll lision diameter. In all of our simulations we have set $\epsilon=\mathrm{k} T$ and the collision diameter equal to the bead diameter, $d^{\prime}=a$. The excluded volume force due to the bounding walls is directed normal to the walls and is also modeled as a truncated Lennard-J ones potential.

The first bead in a chain, bead 1, is located at the bottom surface and does not change position during the course of the simulation (i.e., the chains are permanently tethered). The tethering constraint force, $\mathrm{F}_{\mathrm{i}}^{\mathrm{t}}$, is derived by setting $\mathrm{r}_{\mathrm{i}}^{\mathrm{l}}(\mathrm{t}+\delta \mathrm{t})$ $=r_{i}^{1}(t)$. This directly leads to

$$
F_{i}^{t, 1}=-\left\langle v_{i}\left(r_{i}^{1}\right)\right\rangle-F_{i}^{b r, 1}-F_{i}^{e v, 1}-T^{1} u_{i}^{1}
$$

Note $F_{i}^{t, 1}$ is the force exerted by the wall on the polymer. The polymer exerts a force of equal magnitude and opposite sign on the wall, $-F_{i}^{t, 1}$. We do not need to include the wallexcluded volume force, $F_{2}^{\text {wall, }, 1}$, in eq 7 because bead 1 can never move and hence never penetrate the wall.

An iterative scheme $\mathrm{e}^{42}$ is used to calculate the trajectories of the chains in our simulations. We have previously shown that this scheme is equival ent to a midpoint algorithm. ${ }^{39}$ In the remainder of the discussion we have made lengths dimensionless with the bead diameter $a$, time with the characteristic diffusion time of a bead $\xi \mathrm{a}^{2} / \mathrm{kT}$, and stress with $\mathrm{kT} / \mathrm{a}^{3}$.

The simulation cell has dimensions of $L_{1} \times L_{2} \times L_{3}$ where $L_{1}, L_{2}$, and $L_{3}$ are the lengths in the "1,2,3" directions respectively. The bounding surfaces are parallel to the " $1-3$ " plane and $L_{2}$ is then the gap thickness. To simulate an infinite planar brush, we use periodic boundary conditions in the " 1 " and " 3 " directions. Additionally, we use cell structures and linked lists ${ }^{44}$ to greatly reduce the computational time associated with calculating the excluded volume forces. The number of chains per simulation cell was set equal to 20, and we simulated brushes of lengths $\mathrm{N}=20$ and $\mathrm{N}=40$. We performed a few additional simulations with 40 chains to verify that the results did not significantly change. The dimensionless areal fraction of chains, $\sigma$, is given as $\sigma=\left(\mathrm{N}_{c} \mathrm{a}^{2}\right) /\left(\mathrm{L}_{1} \times\right.$ $\left.\mathrm{L}_{3}\right)$.

To generate the initial chain configurations, the first bead in each chain is randomly placed on the " $1-3$ " tethering plane at $r_{2}^{1}=0.5$. The tethering of the center of the bead ensures that the edge of the bead does not intersect the bottom surface. To generate the remaining beads in the chain, we used one of two methods. For dilute brushes at small areal fractions, the beads positions were specified by a self-avoiding random walk. For larger areal fractions, the chains were initially given straight configurations directed normal to the grafting surface. After generating the initial chain configurations, the simulation was run for an equilibrium period during which time the solvent velocity was set to zero. Compressed equilibrated brushes were created by first generating an equilibrated uncompressed brush and then slowly moving the top wall to 
the desired height. The simulation was then run for another equilibration period. Further details of the brush generation method can be found in ref 46 .

2.1. Calculating the Solvent Velocity in the Brush. The flow is created via the movement of the top plate with a velocity, U. In the absence of the tethered layer a simple linear velocity profile would result; however, due to the brush the flow profile will drastically change. To model the flow, we introduce the ensemble-averaged solvent velocity at position $x_{i},\left\langle u_{i}\left(x_{i}\right)\right\rangle$. Under Stokes's flow conditions we can write the general equation for the averaged solvent velocity in the brush as

$$
\frac{1}{3 \pi} \frac{\partial^{2}\left\langle u_{i}\right\rangle}{\partial x_{j} \partial x_{j}}-\frac{\partial\langle P\rangle}{\partial x_{i}}+\left\langle f_{i}\left(x_{i}, t ; u_{i}\right)\right\rangle=0
$$

In eq $8\langle P\rangle$ is the averaged solvent pressure and $\left\langle\mathrm{f}_{\mathrm{i}}\right\rangle$ is the averaged force density exerted by the brush on the solvent. We note the factor of $3 \pi$ in eq 8 arises because the vel ocity is made dimensionless with $\xi \mathrm{a}^{2} / \mathrm{kT}$ instead of $\mu \mathrm{a}^{3} / \mathrm{kT}$. The averaged force density $\left\langle\mathrm{f}_{\mathrm{i}}\right\rangle$ is equal to $\left\langle\rho\left(\mathrm{X}_{\mathrm{i}}\right) \mathrm{F}_{\mathrm{i}}^{\mathrm{h}}\right)$ where $\rho\left(\mathrm{X}_{\mathrm{i}}\right)$ is the bead density. We calculate $F_{i}^{h}$ using eq 2 . Of course $\left\langle f_{i}\right\rangle$ is a functional of the solvent velocity since the dynamics are governed by the solvent flow. This self-consistent mean-field approach for related Brinkman problems has been shown to be a very good approximation. ${ }^{45}$

In our simulation geometry the averaged solvent velocity reduces to a single scalar velocity component, $\mathrm{u}_{1}\left(\mathrm{x}_{2}\right)$ and is equal to $U$ at $x_{2}=L_{2}$, on the top plate, and $u(0)=0$. The flow strength is characterized by the Weissenberg number, Wi, which we define as equal to the gap average shear rate times the relaxation time for a free chain (i.e., $\mathrm{Wi}=\mathrm{U} \tau_{1} / \mathrm{L}_{2}$. We have shown in a previous publication ${ }^{39}$ that $\tau_{1}=0.0142 \mathrm{~N}^{2}$. The averaged force density $\left\langle\mathrm{f}_{\mathrm{i}}\right\rangle$ has two components, $\left\langle\mathrm{f}_{\mathrm{i}}\right\rangle$ and $\left\langle\mathrm{f}_{2}\right\rangle_{\text {, }}$ which are only functions of $\mathrm{x}_{2} .\left\langle\mathrm{f}_{2}\right\rangle$ serves to alter the solvent pressure and $\left\langle\mathrm{f}_{\mathrm{i}}\right\rangle$ serves to alter the convective velocity field $u_{1}\left(x_{2}\right)$. Since the force densities are only functions of $x_{2}$, we also take averages over the " $1-3$ " plane.

The force densities calculated in the Brownian dynamics simulations contain large fluctuations and very large ensembles are needed to obtain smooth results, ensembles much larger than 20 chains. In steady shear flow we can evoke the ergodic hypothesis and average over time, in addition to averaging over ensembles, to attain better statistics. We can circumvent averaging of the forces in steady shear flow by realizing that the averaged force $\left\langle f_{1}\left(x_{2}\right)\right\rangle$ is equal to $\left\langle u_{1}\left(x_{2}\right) \rho\right.$ $\left.\left(x_{2}\right)\right\rangle$ and $\left\langle f_{2}\left(x_{2}\right)\right\rangle=0$. The force densities assumptions are a direct consequence of the fact that the average velocity of a bead in the " 1 " and " 2 " directions is zero. The averaged equation of motion for the velocity then takes the familiar Brinkman ${ }^{21}$ form for flow through a porous medium:

$$
\frac{1}{3 \pi} \frac{\partial^{2}\left\langle u_{1}\right\rangle}{\partial x_{2} \partial x_{2}}-\left\langle\rho\left(x_{2}\right) u_{1}\right\rangle=0
$$

The corresponding pore size which determines the degree of penetration of the solvent velocity into the brush is equal to $\rho^{-1 / 2}$. The polymer brush though is a dynamic porous medium in that the bead density and hence pore size are complicated functions of solvent velocity and distance from the tethered surface. We have confirmed that $\left\langle\mathrm{f}_{1}\left(\mathrm{x}_{1}\right)\right\rangle=\left\langle\mathrm{u}_{1}\left(\mathrm{x}_{2}\right) \rho\left(\mathrm{x}_{2}\right)\right\rangle$ and $\left\langle\mathrm{f}_{2^{-}}\right.$ $\left.\left(\mathrm{x}_{1}\right)\right\rangle=0$ elsewhere. ${ }^{46}$ Other authors have invoked the Brinkman equation in theoretical approaches ${ }^{15,18,20,26}$ and MonteCarlo simulations ${ }^{31,33}$ of brushes, and also in studying single tethered chains via Brownian dynamics. ${ }^{23}$

\section{Results and Discussion}

3.1. Equilibrium Chain Dimensions. Before discussing the shearing of polymer brushes, we define a measure of the equilibrium brush thickness, $h_{\text {ext. }}$. Following Murat and Grest, ${ }^{47} \mathrm{~h}_{\text {ext }}$ is defined as the distance from the tethering surface at which the bead-density

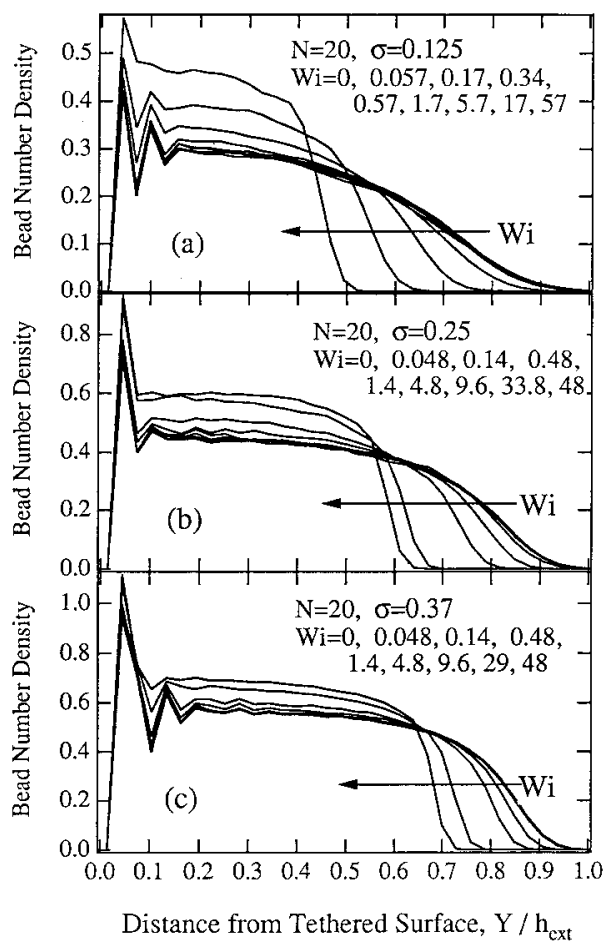

Figure 1. Bead number density versus distance from a tethered surface, $\mathrm{Y} / \mathrm{h}_{\text {hext }}$ for $\mathrm{N}=20$ for a range of Wi at areal fractions of (a) $\sigma=0.125$, (b) $\sigma=0.25$, and (c) $\sigma=0.37$.

Table 1. Equation Brush Thickness

\begin{tabular}{lll}
\hline $\mathrm{N}$ & \multicolumn{1}{c}{$\sigma$} & $\mathrm{h}_{\text {ext }}$ \\
\hline 20 & 0.125 & 13.3 \\
20 & 0.25 & 15.1 \\
20 & 0.37 & 16.8 \\
40 & 0.125 & 24.4
\end{tabular}

profile is equal to zero. $h_{\text {ext }}$ corresponds to the height at which a slight decrease in the gap size will result in a finite normal force. Brushes in which the plate separation is less than $h_{\text {ext }}, L_{2}<h_{\text {ext }}$, will be referred to as compressed brushes. The numerical values for $\left\langle\mathrm{h}_{\mathrm{ext}}\right\rangle$ are given in Table 1 for reference.

3.2. Steady Shear. 3.2.1. Uncompressed Tethered Chains. In this section we discuss the steady shear of uncompressed tethered chains, $L_{2} / h_{\text {ext }}=1.13$. In the uncompressed brushes we are most interested in the physical and hydrodynamic thickness, and the orientation of the chains as a function of $\mathrm{N}, \sigma$, and Wi.

We can best examine the physical thickness of the brush by monitoring the bead density profile. In Figure 1 we have plotted the bead density profiles for $\mathrm{N}=20$ chains at $\sigma=0.125,0.25$, and 0.37 and $\mathrm{Wi}=0-57$. The equilibrium $(\mathrm{Wi}=0$ ) density profiles become more blunt with increasing $\sigma$. For $\sigma=0.125$ the density profiles do not differ from the equilibrium profile until $\mathrm{Wi}=1.7$. At larger Wi the brush physically thins via a decrease in the diffuse tail region of the profile, until at $\mathrm{Wi}=57$ the bead density is nearly a step profile (cf. Figure la). For larger areal fractions of $\sigma=0.25$ and 0.37 , in Figure $\mathrm{I}$, parts $\mathrm{b}$ and $\mathrm{c}$, respectively, physically thinning of the brush is not observed until $\mathrm{Wi}=4.8$. Furthermore, at the largest Wi the brush at the smallest areal fraction shows the most substantial change in thickness, nearly a 50\% decrease in thickness, whilethe most dense brush is approximately $70 \%$ of its original thickness.

A quantitative measure of the thickness of the brush is the hydrodynamic thickness, $L_{h}$. In Figure $2 a$ we 

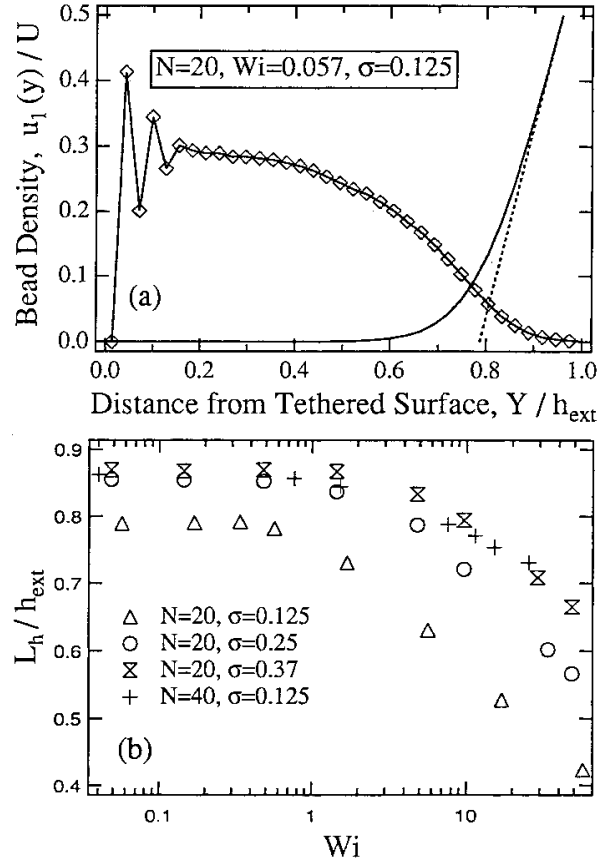

Figure 2. (a) Bead number density (symbols) and velocity (solid line) versus distance from tethered interface, $\mathrm{Y} / \mathrm{h}_{\text {hext }}$ for $\mathrm{N}=20, \mathrm{Wi}=0.057$, and $\sigma=0.125$. The dashed line is the extrapolation of the far-field linear profile. The x-intercept of the extrapolation defines the hydrodynamic thickness, $L_{h}$, of the brush. (b) Hydrodynamic thickness versus Wi.

show the bead density and velocity profile for one case, $\mathrm{N}=20, \mathrm{Wi}=0.057$, and $\sigma=0.125$. The extrapolation of the far-field linear region of the velocity profile to the $x$-axis defines the hydrodynamic thickness of the brush. The vel ocity penetrates only a small portion of the brush and decreases rapidly inside the brush. The same farfield velocity profile in Figure $2 a$ can be attained by replacing the brush with a solid surface of thickness $L_{h}$. The hydrodynamic thickness is smaller than the physical thickness of the brush due to the penetration of the solvent into the brush. It is clear from Figure $2 a$ that the velocity inside the brush does not resemble the linear shear flow profile that would result if the brush were absent.

In Figure $2 b$ the hydrodynamic thickness divided by $h_{\text {ext }}$ versus $\mathrm{Wi}$ is shown. At small $\mathrm{Wi}, \mathrm{L}_{h} / \mathrm{h}_{\text {ext }}$ increases with increasing $\sigma$ for $\mathbf{N}=20$. Also, for an areal fraction of $\sigma=0.125$ the ratio $\mathrm{L}_{h} / \mathrm{h}_{\text {ext }}$ increases when the chain size is doubled from $N=20$ to $N=40$. Both these trends are due to the decreasing tail region in the density profile which decreases with increasing areal fraction and chain length. 48 For $\mathrm{N}=20$ and $\sigma=0.125$ the hydrodynamic thickness decreases for $\mathrm{Wi} \geq 1$.7. The initial decrease in $L_{h} / h_{\text {ext }}$ shifts to larger Wi as the areal fraction increases or as the chain length increases. At large Wi the hydrodynamic thickness shows a powerlaw dependence on $\mathrm{Wi}$ and is a weaker function of $\mathrm{Wi}$ for increasing $\sigma$ and $\mathrm{N}$.

The chains in the tethered layer are stretched and oriented in the " 1 " direction by the flow. We have calculated a measure of the chain tilt: ${ }^{31}$

$$
\langle\theta\rangle=\left\langle\operatorname{Arccos}\left(r_{1}^{c m} /\left|r_{i}^{c m}\right|\right)\right\rangle
$$

where $r_{1}^{\mathrm{cm}} /\left|r_{i}^{\mathrm{cm}}\right|$ is the " 1 " component of the unit vector directed from the chain-tethering point to the chain center of mass and $90-\theta$ is the angle of inclination

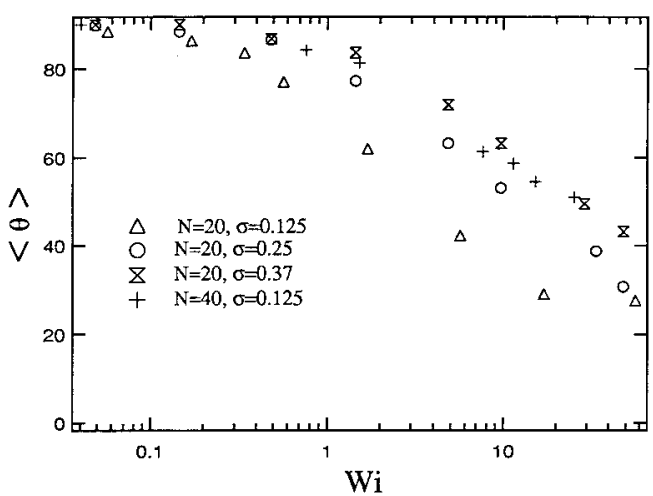

Figure 3. Chain tilt, $\langle\theta\rangle$, versus Wi.
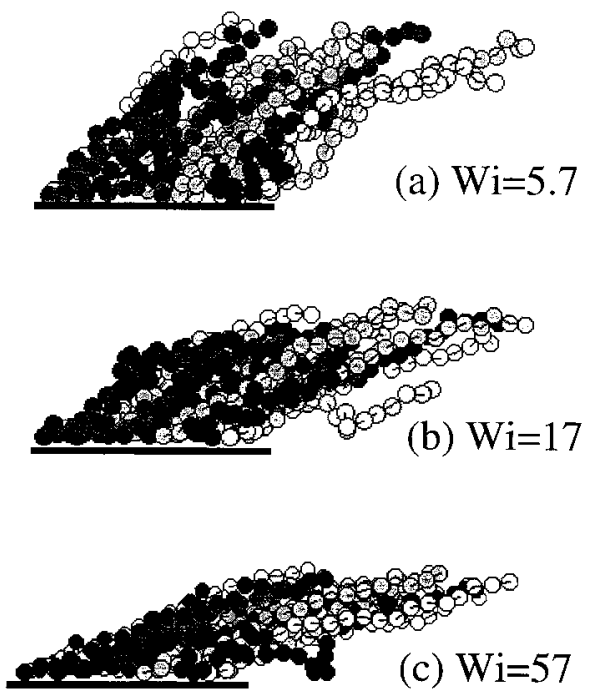

Figure 4. Sample tethered chain configurations for $N=20$ and $\sigma=0.125$ at (a) $\mathrm{Wi}=5.7$, (b) $\mathrm{Wi}=17$, and (c) $\mathrm{Wi}=57$.

from the " 2 " axis. A smaller value of $\langle\theta\rangle$ corresponds to a larger chain tilt or inclination. In Figure 3 we show the dependence of $\langle\theta\rangle$ on $\mathrm{Wi}$. The chains tilt away from the " 2 " axis with increasing Wi. At a fixed Wi, the chains at a higher areal fraction and larger $\mathrm{N}$ shows smaller tilt. It is interesting to compare the decrease in $\langle\theta\rangle$ to the bead density profiles in Figure 1 . The chain tilt becomes appreciable before a change in the bead density profiles is detected. For instance, in Figure 1 a for $\mathrm{N}=20$ and $\sigma=0.125$ at $\mathrm{Wi}=0.57$ the density profile does not differ substantially from the equilibrium profile while in Figure $3\langle\theta\rangle=78^{\circ}$. The degree of inclination of the chains can also be observed in sample configurations of the tethered chains. In Figure $4 a-c$ we show sample chain configurations for $\mathbf{N}=20$ and $\sigma=0.125$ at varying Wi. As the Wi increases, the chains are stretched and the layer thickness decreases. We see in Figure 4c that, even at large flow strengths, $\mathrm{Wi}=57$, not all of the chains are fully stretched.

Theoretical studies differ as to their predictions of the response of a tethered layer to increasing shear rate. Rabin and Alexander ${ }^{13}$ predict that the height will not change while Barrat ${ }^{14}$ predicts the height will increase as the chains are stretched and the screening of the excluded volume interactions decreases. Both theories consider the balance of the osmotic, shear, and elastic forces on the brush; however, they differ in their derivation of the osmotic force. Rabin and Alexander ${ }^{13}$ derive an osmotic force by dividing the excluded vol ume free energy by the brush height while Barrat ${ }^{14}$ takes 


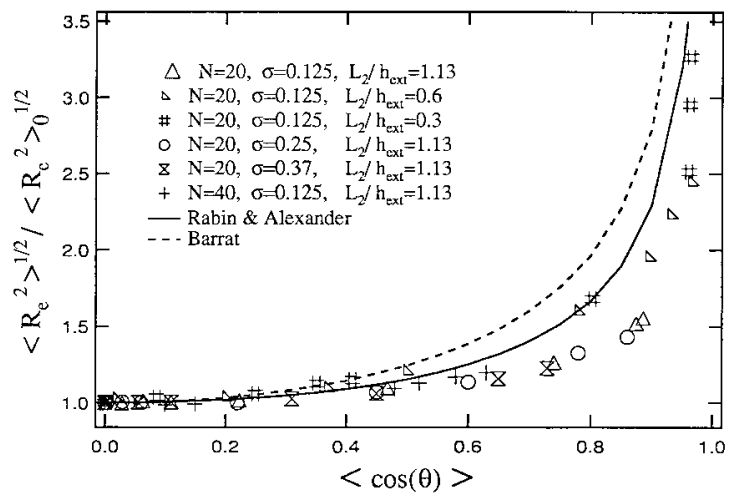

Figure 5. Comparison of chain stretch and inclination to the predictions of Rabin and Alexander ${ }^{13}$ and Barrat ${ }^{14}$.

the derivative of the free energy. We find no evidence of brush thickening, in agreement with the theory of Rabin and Alexander. ${ }^{13}$ Rabin and Alexander ${ }^{13}$ derive a relationship between the chain length and inclination $\left\langle\mathrm{R}_{\mathrm{e}}{ }^{2}\right\rangle\left\langle\left\langle\mathrm{R}_{\mathrm{e}}{ }^{2}\right\rangle_{0}=1 /\left[1-\langle\cos (\theta)\rangle^{2}\right]\right.$ while Barrat ${ }^{14}$ predicts $\left\langle\mathrm{R}_{\mathrm{e}}^{2}\right\rangle /\left\langle\mathrm{R}_{\mathrm{e}}{ }^{2}\right\rangle_{0}=\left[1+\langle\cos (\theta)\rangle^{2}\right]^{2 / 3} /\left[1-\langle\cos (\theta)\rangle^{2}\right]$ where $\mathrm{R}_{\mathrm{e}}$ is the chain end-to-end distance and the subscript 0 denotes an equilibrium property. In Figure 5 we show $\left\langle\mathrm{Re}_{\mathrm{e}}^{2}\right\rangle^{1 / 2} /\left\langle\mathrm{R}_{\mathrm{e}}{ }^{2}\right\rangle^{1 / 2}$ versus $\langle\cos (\theta)\rangle$ for both compressed and uncompressed brushes. We will defer a discussion of the compressed brush results until the following section. The data for the uncompressed brushes lie below both theories but follow the same trend (i.e., the chain extension increases with increasing inclination). Compared to either theory, our simulations indicate less stretch in the chains for a given inclination. This is due to the increased nonlinear el astic restoring force as the chains are extended to near full extension. For this reason, the data for larger chains with $\mathrm{N}=40$ are closer to the theory of the Rabin and Alexander than for $\mathrm{N}=$ 20. Monte Carlo ${ }^{31}$ studies and recent Brownian dynamics ${ }^{38}$ simulations of freely draining brushes subject to a uniform flow field data also fall below the theory of Rabin and Alexander. Recent molecular dynamics simulations ${ }^{37}$ were in agreement with the theory of Barrat for $\left\langle\mathrm{R}_{\mathrm{e}}{ }^{2}\right\rangle^{1 / 2} /\left\langle\mathrm{R}_{\mathrm{e}}{ }^{2}\right\rangle^{1 / 2}$ versus $\langle\cos (\theta)\rangle$, though no brush thickening was reported.

The initial difference in the amount of layer deformation at a given Wi for varying $\sigma$ and $\mathrm{N}$ is attributed to the differing degrees of solvent penetration i.e., the difference in the ratio $L_{h} / h_{\text {ext }}$. The solvent penetration increases with decreasing $\sigma$ and decreasing $\mathrm{N}$ (cf. Figure $2 b$ ). All the brushes have similar values of $L_{h} / h_{\text {ext }}$ at low Wi in Figure 2, except the brush with $\mathrm{N}=20$ and $\sigma=0.125$. This system $(\mathrm{N}=20$ and $\sigma=0.125)$ also shows deformation at much smaller Wi than all the other systems (cf. Figures 1-3). At large Wi, Wi $\gg 1$, the brush thickness decreases and hence the bead density also increases. With increasing $\sigma$ there is a greater resistance to compression, resulting in a slower decrease in the brush thickness and $L_{h}$.

Previous simulations have examined the deformation of an uncompressed polymer brush in shear flow. 31-34,37,38 Monte Carlo simulations by Lai and Binder ${ }^{31}$ and Miao et al. ${ }^{33}$ for weak flows covering a decade of shear rates found no appreciable change in brush height. Lai and Lai 32 simulated larger shear rates (about 6 times the largest shear rate in Lai and Binder's simulations) and found no appreciable change in the brush thickness. Molecular dynamics simulations by Grest ${ }^{37}$ showed a decrease in the perpendicular component of the radius
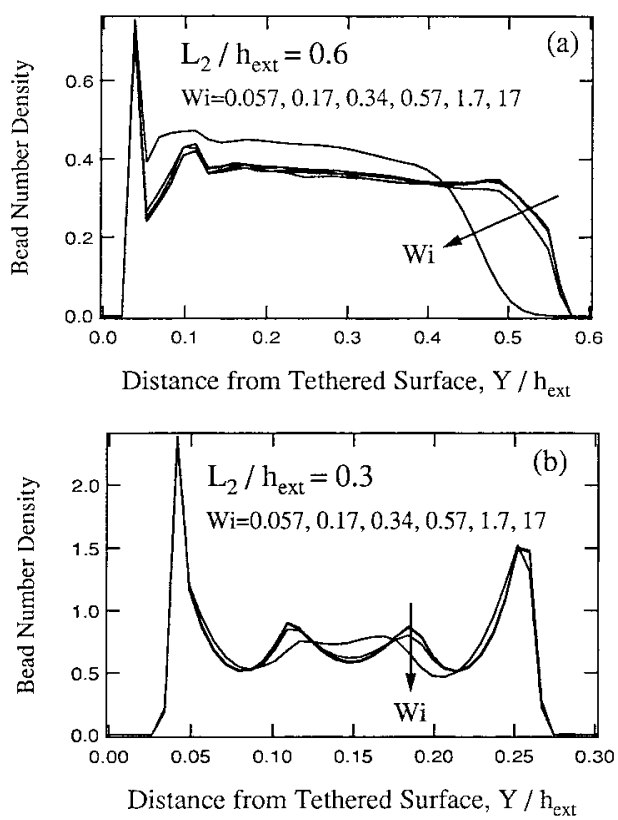

Figure 6. Bead number density versus distance from a tethered surface, $\mathrm{Y} / \mathrm{h}_{\text {hext }}$ for $\mathrm{N}=20$ and $\sigma=0.125$ at compressions of (a) $L_{2} / h_{\text {ext }}=0.6$ and (b) $L_{2} / h_{\text {ext }}=0.3$.

of gyration at the largest shear rates to a value of approximately $65 \%$ the equilibrium value for a system with $\mathbf{N}=100$ and $\sigma=0.03$. Molecular dynamics of smaller chains $(\mathrm{N}=20)$ at large surface coverages $(\sigma=$ 0.33 ) by Peters and Tildesl ey ${ }^{34}$ showed a decrease in the perpendicular component of the radius of gyration upon shear to approximately $70-80 \%$ the equilibrium value. The brush height decreased slightly at the largest shear rates in both the work of Grest ${ }^{37}$ and of Peters and Tildesley. ${ }^{34}$ More recently, molecular dynamics simulations by Neelov et al. ${ }^{38}$ of a freely draining brush (with $\mathrm{N}=51$ and 101) showed shear thinning of the brush thickness of up to $50 \%$. In the simulations presented in this paper a brush decreases in thickness at large Wi to between 50 and $75 \%$ the equilibrium height. All the previously mentioned simulations agree that the largest deformation of the brush occurs in the shear direction, and when layer thinning is observed, it occurs at very large Wi. The varying degrees of brush thinning (or lack of) observed in the different simulations is primarily due to the different range of shear rates and chain lengths studied. In the current study we simulated small chains and very large Wi (covering 3 decades in magnitude) which are both reasons for the substantial brush thinning observed. We note that our limited data for larger chains of $\mathrm{N}=40$ in Figure $2 \mathrm{~b}$ show less shear thinning than a brush with $\mathrm{N}=20$ at the same surface coverage.

3.2.2. Compressed Tethered Chains. In this section we discuss the steady shear of compressed brushes where $L_{2} / h_{\text {ext }}<1$. We have limited our studies to one areal fraction, $\sigma=0.125$, and chain lengths of $\mathrm{N}$ $=20$. Unless otherwise stated, all results will be presented for these parameters with varying Wi and gap thickness, $L_{2} / h_{\text {ext }}$.

In Figure 6 we show bead density profiles for brushes compressed to $L_{2} / h_{\text {ext }}=0.6$ and $L_{2} / h_{\text {ext }}=0.3$. At small Wi the density profile for $L_{2} / h_{\text {ext }}=0.6$ looks similar to the profiles in Figure 1 for the uncompressed brushes at $\sigma=0.25-0.37$. The density profile for $\mathrm{L}_{2} / \mathrm{h}_{\text {ext }}=0.3$ has distinct peaks associated with ordering of the 


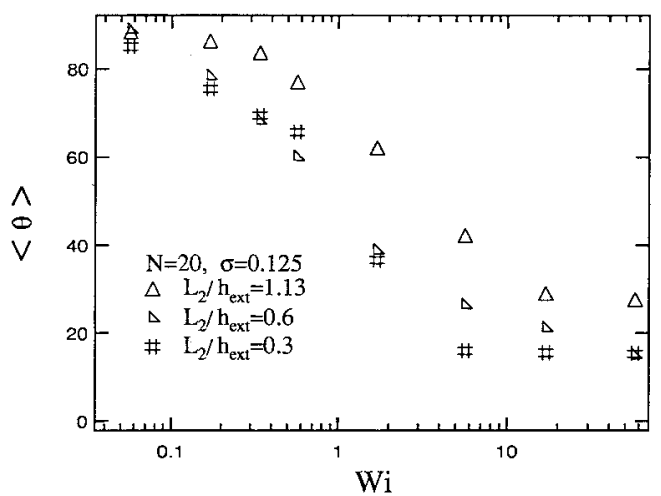

Figure 7. Chain tilt, $\langle\theta\rangle$, versus Wi.

compressed chains. The mean volume fraction of the chains in the compressed brush at $L_{2} / h_{\text {ext }}=0.3$ is equal to 0.33 , well-below the close-packed limit for a system of spheres. ${ }^{41}$ Furthermore, the peaks are asymmetric and larger near the tethered surface, as would be expected since the density profiles in the uncompressed brushes indicate some ordering near the tethering surface (cf. Figure 1). The density profiles for both brushes in Figure 6 change very little for $\mathrm{Wi} \leq 1.7$. At $\mathrm{Wi}=17$ the brush at $L_{2} / h_{\text {ext }}=0.6$ decreases in thickness and there is a diffuse tail region in the density profile. We recall that, for the uncompressed brushes, shear flow tended to decrease the tail region in the density profile. At $\mathrm{Wi}=17$, the density profile for the brush at $\mathrm{L}_{2} / \mathrm{h}_{\text {ext }}$ $=0.3$ changes very little near the walls, while the peaks that existed at lower $\mathrm{Wi}$ in the center of the gap diminished as shown in Figure 6b. The shear flow thus tends to decrease the ordering which was present in the center of the gap at smaller Wi.

To quantitatively measure the brush deformation, we show the dependence of $\langle\theta\rangle$ on Wi in Figure 7 for an uncompressed brush and compressed brushes at gap widths of $L_{2} / h_{\text {ext }}=0.3$ and 0.6. The compressed brushes have a larger inclination at a given Wi than the uncompressed brush. For Wi $<0.3$, the most compressed brush, $L_{2} / h_{\text {ext }}=0.3$, shows more deformation than the brush at $L_{2} / h_{\text {ext }}=0.6$. For $0.3<\mathrm{Wi}<1$ the trend changes, and finally for $\mathrm{Wi}<1$ the magnitude of $\langle\theta\rangle$ is slightly smaller for $L_{2} / h_{\text {ext }}=0.3$ than for $L_{2} / h_{\text {ext }}=$ 0.6. The compressed brushes do not show an appreciable decrease in thickness until Wi $>10$ and the decrease is larger as $L_{2} / h_{\text {ext }}$ increases. The decrease in layer thickness is due to the finite length of the chains. At equilibrium, the chains in a compressed brush adopt more compact configurations than those in an uncompressed brush. The sum of the chain extensions in the " 1 " and " 2 " directions are constrained by the chain length. The compressed brush has less extension in the " 2 " direction and thus can be stretched to a greater extent in the "1" direction before the finite chain length becomes a factor.

In Figure 5 we compared the ratio of $\left.\left\langle\mathrm{R}_{\mathrm{e}}^{2}\right\rangle^{\mid / 2} /\left\langle\mathrm{R}_{\mathrm{e}}\right\rangle^{2}\right\rangle^{1 / 2}$ versus $\langle\cos (\theta)\rangle$ for the compressed brushes to the uncompressed brushes and the theories of Rabin and Alexander and Barrat. For small $\langle\cos (\theta)\rangle$ the data for the compressed brushes lie between the Rabin and Alexander ${ }^{13}$ and Barrat ${ }^{14}$ theories and above the uncompressed results. At larger $\langle\cos (\theta)\rangle$ the compressed brush data line lies below both theories. The better agreement between the compressed brush data and the theories verses the uncompressed brush data can be attributed to the fact that the compressed layers can

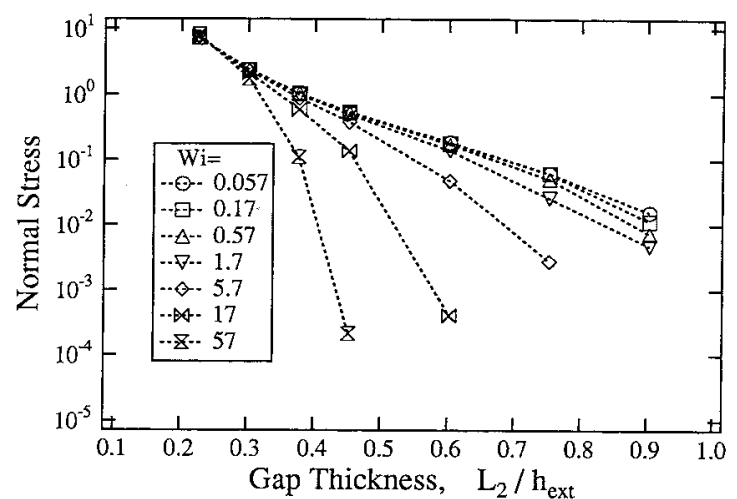

Figure 8. Normal stress versus $L_{2} / h_{\text {ext }}$ for $N=20, \sigma=0.125$ and various values of $\mathrm{Wi}$.

be deformed more (tilted and stretched) before the finite extensibility of the chains becomes important.

3.2.3. Effective Viscosity and Normal Pressure. We define an effective viscosity, $\mu_{\text {eff, }}$ for the layer as the shear stress on either the tethering surface or top moving surface divided by the gap average shear rate, $\mathrm{U} / \mathrm{L}_{2}$. I $\mathrm{n}$ the absence of the tethered polymer layer the effective viscosity is equal to the solvent viscosity. In our model the plates are homogeneous (i.e., they do not include the atomic structure of the surface), and hence a polymer can only transmit a shear stress to a tethering plate via the tethering force. Any additional shear stress on the surfaces is due to the solvent and is therefore proportional to the local shear rate at the surface. Note the polymer indirectly contributes to the solvent shear stress since the bead-density profile and the vel ocity profile are solved self-consistently. We also calculate the normal force on both the top and bottom plate due to the polymer chains. A normal stress (or normal pressure) is defined as the average normal force on the surface divided by the area of the surface. The total force exerted by the fluid on the brush is transmitted through the chains to the tethering surface, and this results in equal stresses at the top and bottom surface, ${ }^{46}$ as would be expected.

In Figure 8 we show the normal stress versus $L_{2} / h_{\text {ext }}$ for $\mathrm{N}=20, \sigma=0.125$, and $\mathrm{Wi}=0.057-57$. At $\mathrm{Wi}=$ 0.057 the normal stress was found to be equal to the equilibrium normal stress for all $L_{2} / h_{\text {ext }}$. The equilibrium normal stress increases with decreasing $L_{2} / h_{\text {ext }}$ due to the osmotic compressibility of the brush. ${ }^{30,49-51} \mathrm{~F}$ or $L_{2} / h_{\text {ext }} \geq 0.3$, the normal stress at a fixed gap width $L_{2} /$ $h_{\text {ext }}$ decreases with increasing Wi. For $L_{2} / h_{\text {ext }}=0.23$ the normal stress did not change over the range of Wi simulated. Furthermore, as the gap width increases, the initial decrease of the normal stress occurs at a smaller Wi. The polymer transmits a normal stress to the walls through the excluded volume forces. A finite normal stress only results if the chains tethered to the bottom surface come into contact with the top surface. In section 3.2.2. we demonstrated that the physical thickness of the tethered layers decreases with increasing $\mathrm{Wi}$ and that the decrease at a given $\mathrm{Wi}$ is larger with increasing $\mathrm{L}_{2} / \mathrm{h}_{\text {ext }}$. The same trend in the physical thickness of the brushes presented in section 3.2.2. is observed in the normal stress in Figure 8. We find no evidence of normal stress increasing with Wi.

In Figure 9 we show the effective viscosity, made dimensionless with the solvent viscosity, versus $L_{2} / h_{\text {ext }}$ for $\mathrm{N}=20, \sigma=0.125$, and $\mathrm{Wi}=0.057-57$. At a given gap width, the viscosity does not change with increasing 


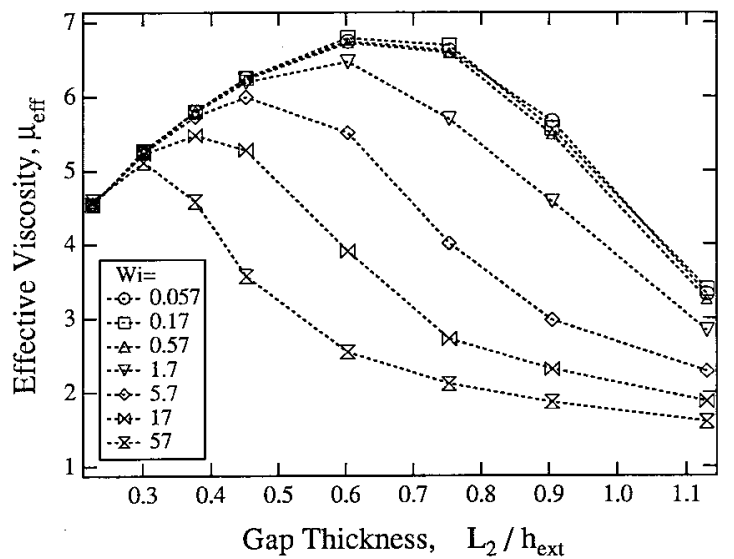

Figure 9. Effective brush viscosity versus $L_{2} / h_{\text {ext }}$ for $N=20$, $\sigma=0.125$, and various values of Wi.

Wi for $\mathrm{Wi}<1.7$ and this corresponds to the zero shear brush viscosity. The zero shear brush viscosity first increases with decreasing gap width until the brush is compressed to $60 \%$ of the original uncompressed brush thickness and attains a maximum value of nearly 7 . At smaller gap widths the zero shear viscosity decreases slowly with decreasing gap width. As the Wi is increased at a given gap width, the brush viscosity decreases. For the two smallest gap widths simulated, $L=0.23$ and 0.3 , the effective viscosity changes very little with increasing Wi.

The trends in the effective viscosity can be understood by revisiting the Brinkman equation:

$$
\frac{1}{3 \pi} \frac{\partial^{2}\left\langle\mathrm{v}_{1}\right\rangle}{\partial \mathrm{x}_{2} \partial \mathrm{x}_{2}}-\left\langle\rho\left(\mathrm{x}_{2}\right) \mathrm{v}_{1}\left(\mathrm{x}_{2}\right)\right\rangle=0
$$

The shear rate on the top plate can be estimated by dividing the velocity on the top plate, $U$, by the characteristic distance over which the velocity decays, or the pore size, $\kappa$. The pore size in the brush is equal to $\rho\left(X_{2}\right)^{-1 / 2}$. The effective viscosity then scales as $(U / \kappa) /$ $\left(U / L_{2}\right)$ or equivalently $L_{2} / \kappa$. First, we consider the compressed layers at $L_{2} / h_{\text {ext }}<0.6$. If we approximate the compressed layer as a step function of constant density $\rho$, then $\rho \sim \sigma / L_{2}$ and the effective viscosity $\sim\left(\mathrm{L}_{2} \sigma\right)^{1 / 2}$. In Figure 10a we show a semilog plot of the zero shear effective viscosity divided by $\sigma^{1 / 2}$ versus $L_{2} /$ $\mathrm{h}_{\text {ext }}$ for $\mathrm{N}=20$ at $\sigma=0.125$ and 0.25 . The data are in agreement with the power law scaling of $\mu_{\text {eff }} \sim\left(\mathrm{L}_{2} / \mathrm{h}_{\mathrm{ext}}\right)^{1 / 2}$ and collapses onto a single curve for $L_{2} / h_{\text {ext }}<0.5$, confirming the scaling $\mu_{\text {eff }} \sim\left(L_{2} \sigma\right)^{1 / 2}$ for small gaps. This scaling will fail when the brush is compressed to a length scale comparable to the persistence length of the chain. For uncompressed brushes, $\left(L_{2} / h_{\text {ext }}\right)>1$, the characteristic distance over which the velocity decays is the $L_{2}-L_{h}$ and the effective viscosity scales as $\mu_{\text {eff }} \sim$ $L_{2} /\left(L_{2}-L_{h}\right)$. In Figure 10b we have graphed $\mu_{\text {eff }}$ versus $L_{2} / h_{\text {ext }}$ and compared the simulations to $L_{2} /\left(L_{2}-L_{h}\right)$. We find very good agreement $L_{2} / h_{\text {ext }} \geq 1$.

In the experiments of Klein et al. ${ }^{2}$ the top plate velocity was held constant as the gap width decreased, resulting in an increasing gap Wi as the gap decreased. In Figure 1la we show results for a simulation in which the top plate vel ocity was held constant at a value of $U$ $=0.15$. This plate vel ocity corresponds to $\mathrm{a} \mathrm{Wi}=0.057$ at $L_{2} / h_{\text {ext }}=1.13$. The shear stress now increases with decreasing gap width and scales as $\left(\mathrm{L}_{2} / \mathrm{h}_{\mathrm{ext}}\right)^{-1 / 2}$ for small gaps (shown as the dashed line in Figure 11a). The

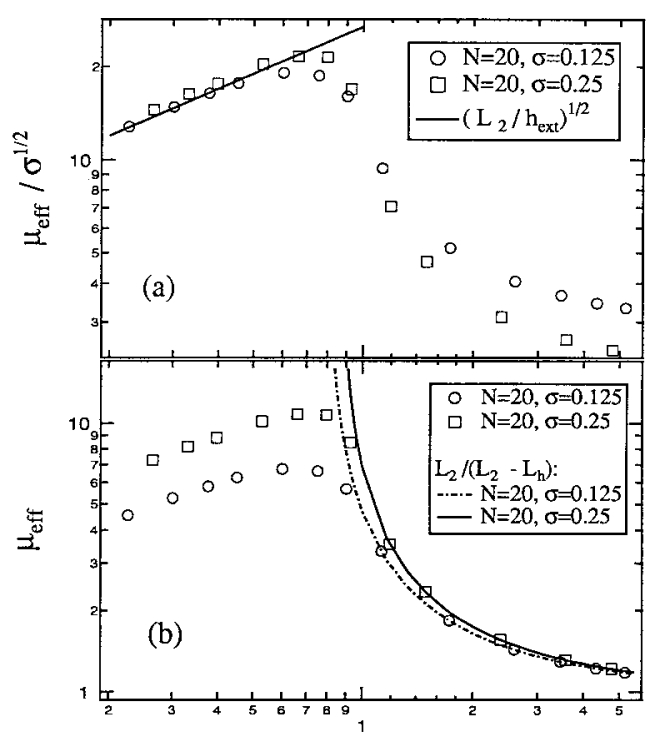

Gap Thickness, $\mathrm{L}_{2} / \mathrm{h}_{\mathrm{ext}}$

Figure 10. (a) Semilog plot of the zero shear brush viscosity divided by $\sigma^{1 / 2}$ versus $L_{2} / h_{\text {ext. }}$. The solid line shows the expected power law $\left(L_{2} / h_{\text {ext }}\right)^{1 / 2}$ for small compressions. (b) Semilog plot of the zero shear brush viscosity versus $L_{2} / h_{\text {ext }}$. The lines are equal to $L_{2} /\left(L_{2}-L_{h}\right)$.
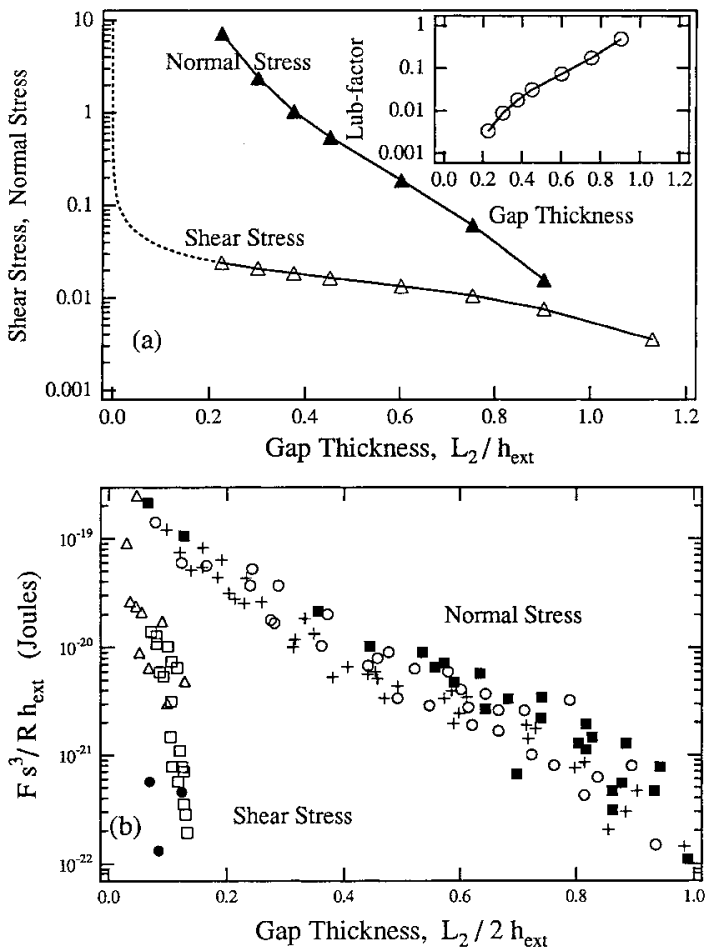

Figure 11. (a) Shear and normal stress versus $L_{2} / h_{\text {ext }}$ for $N$ $=20, \sigma=0.125$, and a constant plate velocity equal to $\mathrm{U}=$ 0.15 . The inset shows the lubrication factor (shear stress divided by normal stress) versus $L_{2} / h_{\text {ext. }}$ (b) Experimental results of Klein et al. ${ }^{2}$ for the shear of mica surfaces bearing polystyrene brushes with molecular weights of $2.6 \times 10^{4}, 1.4$ $\times 10^{5}$, and $3.75 \times 10^{5}$. The experimental stresses are normalized with $h_{\text {ext }} R / s^{3}$ where $R$ is the radii of curvature of the mica sheets in the SFA, $S$ is the mean spacing between chain anchoring points, and $h_{\text {ext }}$ is the brush height.

shear rates simulated in Figure 11a are in the linear viscoel astic regime, and thus the shear stress curves for smaller velocities $(U<0.15)$ will only differ quantitatively by a multiplicative factor, but not qualitatively. The experiments of Klein et al. ${ }^{2}$ are shown in Figure 
11b. In contrast to the experiments, we are able to measure shear stresses for all gap widths; however, this difference is merely due to the inherently different resolution in the simulation and experiment. A more important trend is that the shear forces in both the experiments and simulations are much smaller than the normal forces. The inset of Figure 11 a shows the lubrication factor (ratio of the shear-to-normal stress) versus gap thickness for the same range of parameters shown in Figure 11a. Upon compression a polymer brush is a good lubricant as it supports a large normal load while producing a small shear stress. Furthermore, our scaling theory also predicts a sharp rise in the shear stresses for strongly compressed brushes in qualitative agreement with the experimental data of Klein et al. ${ }^{2}$ We note that although we were unable to attain simulation data for $L_{2} / h_{\text {ext }}<0.2$, the data for $L_{2} /$ $h_{\text {ext }}<0.5$ do scale as $\left(L_{2} / h_{\text {ext }}\right)^{-1 / 2}$ which lends support for our scaling theory.

Recent molecular dynamics simulations by Grest ${ }^{36,37}$ show similar trends for the shear stresses (i.e., increasing upon brush compression and having a magnitude smaller than the normal forces). An important point raised by Grest ${ }^{36,37}$ is that while the normal forces in a plate/plate system (simulation geometry) and a crossed cylinder system (SFA geometry) are directly related, the relationship for the shear forces is unclear.

3.3. Oscillatory Shear. 3.3.1. Velocity Calculation. In the oscillatory shear simulations we now impose a velocity at the top plate, $v_{1}\left(x_{1}=L_{2}\right)=U$ cos$(\omega \mathrm{t})$. The gap-averaged Weissenberg is defined as Wi $=\mathrm{U} / \mathrm{L}_{2} \tau_{1}$. Both the frequency, $\omega$, and time, $\mathrm{t}$, have been made dimensionless with $\tau_{1}$. Since the vel ocity of the top plate is time varying, it follows that the force density in the brush will now also be a function of time.

Though it is straightforward to solve the Brinkman equation, eq 8, at every time step, we cannot simulate large enough ensembles to get accurate force densities. A conservative estimate ${ }^{46}$ of the ensemble size necessary to attain accurate time and spatial resolution of the force densities is 1000 chains which corresponds to approximately a $1 / 3$ year of CPU time.

Due to these computational limitations, we will assume that the force density, $\left\langle\mathrm{f}_{1}\left(\mathrm{x}_{2}\right)\right\rangle$, is zero throughout the brush, giving a simple shear vel ocity profile. This is a poor approximation for small frequencies, as $\omega \rightarrow 0$ we approach the limit of steady shear, but we are most interested in the chain dynamics at high frequencies and large Wi, corresponding to the conditions in the experiments of Klein et al. ${ }^{1}$ As a check of the previous approximation, we have calculated the average of the quantity $f_{1}\left(x_{2}, t\right) v_{1}\left(x_{2}, t\right) /\left|v_{1}\left(x_{2}, t\right)\right|$ where we have averaged over both time and ensembles. $f_{1}\left(x_{2}, t\right) v_{1}\left(x_{2}, t\right) /\left|v_{1}\left(x_{2}, t\right)\right|$ is the projection of the force density $f_{1}\left(x_{2}, t\right)$ in the direction opposing the flow and is thus a measure of the average hydrodynamic resistance of the layer. In fact, we shall refer to the averaged value of $f_{1}\left(x_{2}, t\right) v_{1}$ $\left(x_{2}, t\right) /\left|v_{1}\left(x_{2}, t\right)\right|$ as the hydrodynamic resistance of the layer. We note that, due to the symmetry of the flow, the time average of $f_{1}\left(x_{2}, t\right)$ alone is always zero. In Figure 12 we show the hydrodynamic resistance versus distance from the tethered layer for a compressed brush, $\mathrm{L}_{2} / \mathrm{h}_{\text {ext }}=0.38$, at $\mathrm{Wi}=570$. At $\omega=10$, the resistance is quite large and decreases substantially at the higher frequencies, $\omega=100$ and 200 . The displacement of the brush during the oscillatory cycle is proportional to the strain amplitude which we define as $\mathrm{A}=\mathrm{Wi} / \omega$. At large

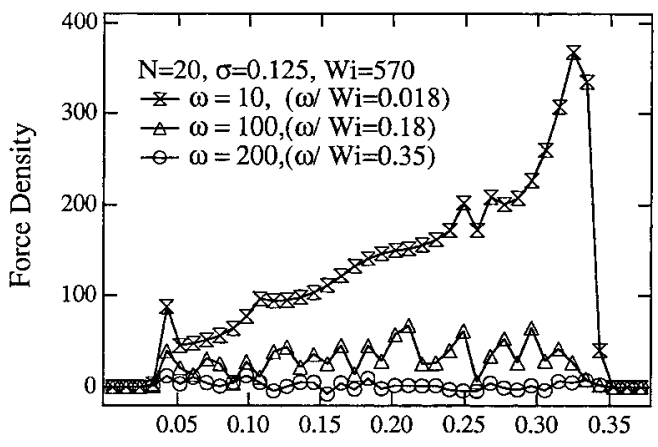

Distance from Tethered Surface, $Y / h_{e x t}$

Figure 12. Averaged force density, $f_{1} v_{1} /\left|v_{1}\right|$, versus distance from a tethered surface for $\mathrm{L}_{2} / \mathrm{h}_{\text {ext }}=0.38, \mathrm{Wi}=570$, and $\omega=$ 10,100 , and 200. Averages have been taken over time and ensembles as described in the text.

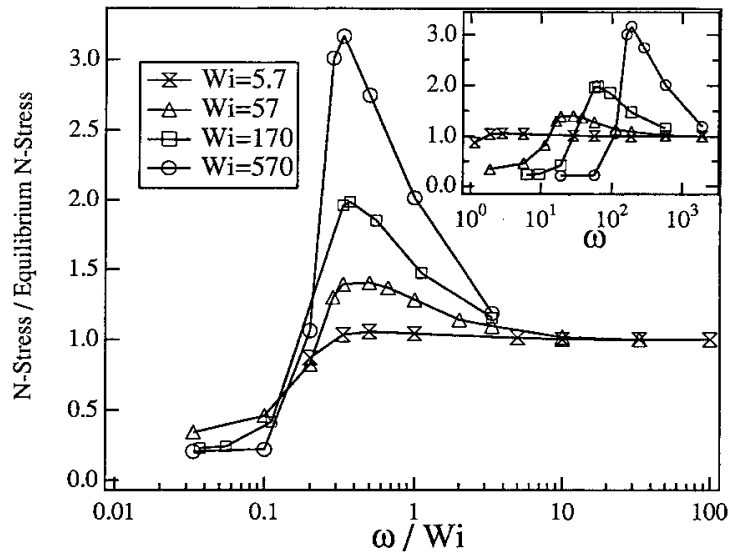

Figure 13. Normal stress divided by the equilibrium normal stress versus frequency divided by Wi for a compressed brush at $\mathrm{L}_{2} / \mathrm{h}_{\text {ext }}=0.38$ and an areal fraction $\sigma=0.125, \mathrm{~N}=20$, and $\mathrm{Wi}=5.7,57.170$, and 570 . The inset shows normal stress divided by the equilibrium normal stress versus frequency.

Wi the beads in a chain move with the local fluid velocity until they are they are constrained by the tethering point. Keeping the strain amplitude small (relative to the chain length) ensures that the chain never "feels" its tethering constraint and the beads move affinely with the fluid, resulting in a small hydrodynamic resistance in the layer. When A is small and Wi large, the brush behaves much like a free suspension of spheres. We will show shortly that the interesting brush dynamics are governed by the strain amplitude and dramatic increases in the normal stress occur in a regime in which the chains are not constrained by their tether. This is the regime in which our assumption of $\left\langle\mathrm{f}_{1}\left(\mathrm{x}_{2}\right)\right\rangle=0$ is most valid. Note that in all of the oscillatory shear simulations presented in this section we have thus made the assumption that $\left\langle\mathrm{f}_{1}\left(\mathrm{x}_{2}\right)\right\rangle=0$. The velocity profile is then given by $v_{1}\left(x_{2}, t\right)=x_{2} W i \cos (\omega t)$. The normal stresses are positive definite over the entire oscillatory cycle. We present values for the normal stresses and bead density profiles which are time and ensemble-averaged. The time averaging is taken over the full duration of the simulation and hence encompasses many oscillatory cycles.

3.3.2. Compressed Tethered Chains. We focus first on the normal stresses for compressed layers. In Figure 13 we show the normal stress (divided by the equilibrium value) versus $1 / \mathrm{A}=\omega / \mathrm{Wi}$ for a compressed brush at $\mathrm{L}_{2} / \mathrm{h}_{\text {ext }}=0.38$ with $\mathrm{N}=20, \sigma=0.125$, and Wi $=5.7-570$. For all Wi there is a critical frequency 


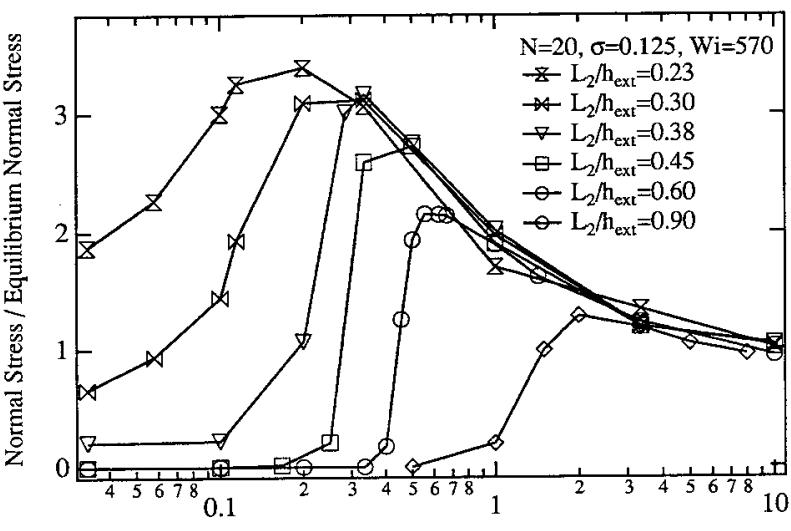

$\omega / \mathrm{Wi}$

Figure 14. Normal stress divided by the equilibrium normal stress versus frequency divided by Wi for compressed brushes at $\mathrm{L}_{2} / \mathrm{h}_{\text {ext }}=0.23-0.9$ and $\mathrm{Wi}=570, \sigma=0.125$, and $\mathrm{N}=20$.

where the normal stress increases and the maximum in the normal stress occurs at approximately the same value of $\omega / \mathrm{Wi}(\omega / \mathrm{Wi}=0.3-0.4$ or $\mathrm{A}=2.5-3)$ for all Wi. The increase in the normal stress can be as large as 3 times the equilibrium value for $\mathrm{Wi}=570$. The critical frequency and the stress at the critical frequency increases with increasing Wi.

In Figure 14 we show the normal stress versus $\omega / \mathrm{Wi}$ for $\mathrm{Wi}=570$ and several gap widths. For all gap widths in Figure 14 there is a critical frequency where the normal stress increases. As the gap width decreases, the critical frequency decreases and the range of frequencies, where the normal stress exceeds the equilibrium value, increases.

The increase in the normal stress is due to the increased collisions beads with their neighbors as they are sheared past each other. The collisions increase the osmotic pressure in the brush which swells the compressed brush and leads to an increase in the normal stress. This is analogous to "shear-induced diffusion" in the theory of free suspensions, ${ }^{52,53}$ where the collision process increases the fluctuating kinetic energy in a suspension and can cause particles in a suspension to diffuse from regions of high concentration to low. 52,53 In Figure 15 we show bead density profiles versus distance from the tethered surface for brushes compressed to $L_{2} / h_{\text {ext }}=0.6$ and $L_{2} / h_{\text {ext }}=0.3$. The frequencies in Figure 15a,b correspond to the frequencies at which the largest increase in normal stress occurred in Figure 14. For both brushes we see a distinctive increase in the bead density profile near the upward surface. The growth in the peak near the upward plate results from the swelling of the brush.

We turn now to a discussion of the trends in the normal stresses observed with increasing Wi in Figure 13 and increasing gap width in Figure 14. The normal stresses decrease toward zero at small frequencies because the flow approaches steady shear, leading to a decrease in the normal stress as a result of a physical thinning of the brush. The layer thinning occurs when a chain is stretched to near full extension. If we assume that the chain ends are at the upward surface, as shown in the schematic in Figure 16, then simple geometric arguments show that this occurs for a displacement $\delta \mathrm{x}$ $=\left(\mathrm{N}^{2}-\mathrm{L}_{2}^{2}\right)^{1 / 2}$. If we assume that the chain end moves affinely with the top plate velocity and traverses from $+\delta \mathrm{x}$ to $-\delta \mathrm{x}$ during half a cycle (i.e., returning to its initial starting point after a full oscillatory cycle), then
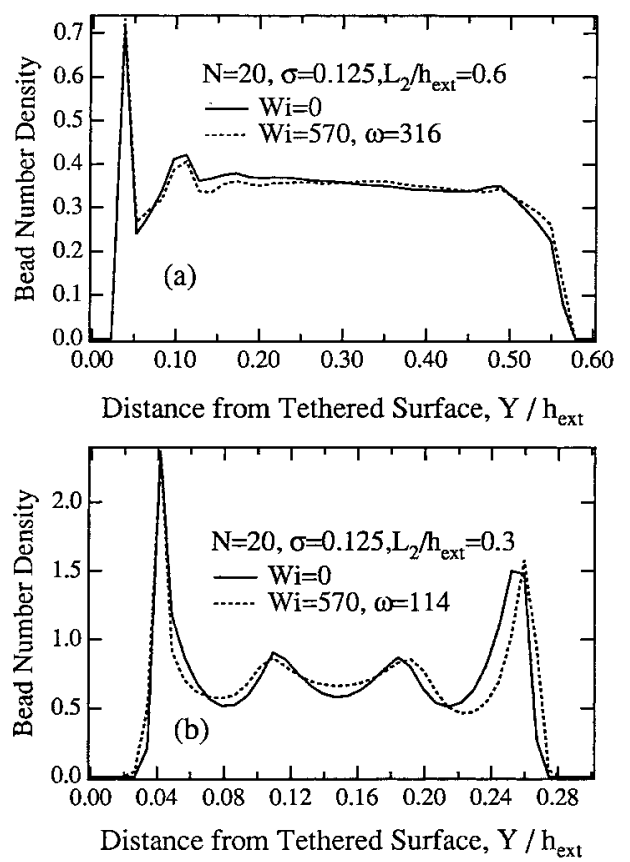

Figure 15. Bead density versus distance from a tethered surface $Y / h_{\text {ext }}$ for brushes compressed to (a) $L_{2} / h_{\text {ext }}=0.6$ and (b) $L_{2} / h_{\text {ext }}=0.3$.

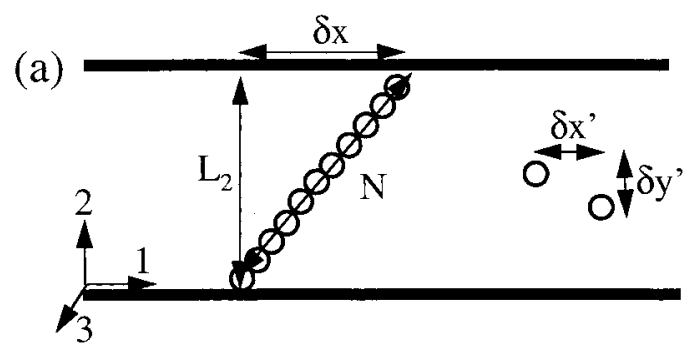

(b)

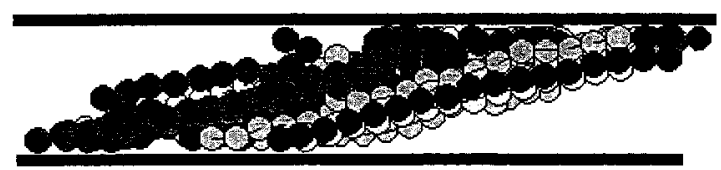

Figure 16. (a) Schematic of a stretched polymer chain under shear flow. (b) Sample configuration of the tethered chains at $\mathrm{L}_{2} / \mathrm{h}_{\text {ext }}=0.38, \mathrm{Wi}=570, \omega=189, \mathrm{~N}=20$, and $\sigma=0.125$.

$\delta \mathrm{x}=\mathrm{L}_{2} \mathrm{Wi} / \omega$. From geometric arguments then

$$
\omega \mathrm{Ni}=\left[\left(\mathrm{N} / \mathrm{L}_{2}\right)^{2}-1\right]^{-1 / 2}
$$

(cf. Figure 16). This is the critical ratio at which the chain will begin to feel its tether point and is the lower limit of $\omega / \mathrm{Wi}$ where an increase in the normal stress will occur. For the parameters in Figure $13(\mathrm{~N}=20, \sigma$ $=0.125$, and $\mathrm{L}_{2} / \mathrm{h}_{\text {ext }}=0.38$ ) eq 12 predicts that thestress will increase at $\omega \mathrm{Mi}=0.26$ in agreement with the simulation data. In Figure $16 \mathrm{~b}$ we show a sample tethered chain configuration under conditions where the largest increase in normal stress was observed in Figure 13: $\mathrm{Wi}=570$ and $\omega=189(\omega / \mathrm{Wi}=0.33)$ at a compression $L_{2} / h_{\text {ext }}=0.38$. The configuration shown in Figure 13 is representative of the maximum chain stretch during a cycle. We see that the chains are stretched to near full extension as expected from the previous arguments. Equation 12 al so predicts that the increase in the stress will occur at a lower ratio of $\omega / \mathrm{Wi}$ 


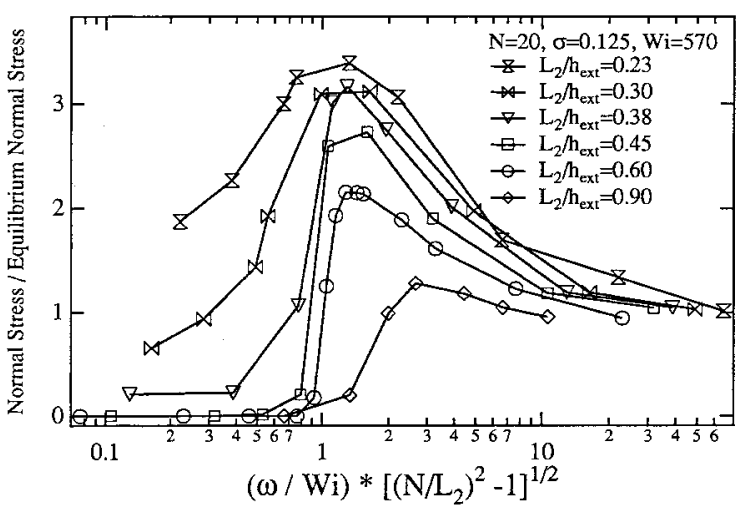

Figure 17. Normal stress divided by the equilibrium normal stress versus rescaled frequency, $(\omega \mathrm{Ni})\left[\left(\mathrm{N} / \mathrm{L}_{2}\right)^{2}-1\right]^{1 / 2}$ for $\mathrm{N}$ $=20, \sigma=0.125$, and $\mathrm{Wi}=570$.

as the gap width increases in accord with the trends observed in Figure 14. In Figure 17 we show the normal stress data from Figure 14 versus $(\omega / \mathrm{Wi})\left[\left(\mathrm{N} / \mathrm{L}_{2}\right)^{2}-1\right]^{1 / 2}$. The simple scal ing presented in eq 17 collapses the data very well such that the maximum stress occurs at approximately a value of $(\omega / \mathrm{Wi})\left[\left(\mathrm{N} / \mathrm{L}_{2}\right)^{2}-1\right]^{1 / 2}=1$. We note that for a brush-brush system we replace $\mathrm{N}$ by $2 \mathrm{~N}$ and $L_{2}$ by $2 L_{2}$ in the previous scalings and recover again eq 12.

In the limit of $(\omega / \mathrm{Wi}) \rightarrow \infty$ the chains do not move during the oscillation and the normal stresses in Figures 13 and 14 approach the equilibrium value. We now calculate the highest frequency at which collisions will occur. We consider two beads which are separated in the " 2 " direction by a distance $\delta y$ ' and in the " 1 " direction by a distance $\delta x^{\prime}$ (cf. Figure 16a). Since the flow is linear, the relative velocity of the beads will be equal to Wi $\delta y^{\prime}$. The maximum relative displacement of the beads in the flow direction during half a oscillatory cycle is $\delta \mathrm{x}^{\prime}=\delta \mathrm{y}^{\prime} 2 \mathrm{Wi} / \omega$. If we assume that the beads are isotropically distributed, then $\delta x^{\prime}=\delta y^{\prime}$ and it follows that $\omega / \mathrm{Wi}=2$. Thus, the upper limit where an increase in the normal stress will occur, $\omega \mathrm{Wi}=2$, is independent of gap width in agreement with the data in Figure 14.

In summary, the critical parameter in determining whether increases in the normal forces occur during oscillation at large Wi is the strain amplitude. The smallest amplitude at which increases occur is when the brush is oscillated on the order of the Kuhn length where beads first begin to collide during an oscillatory cycle. The largest amplitude at which thickening occurs is dictated by the finite extensibility of the polymer chains. The magnitude of the largest amplitude is on the order of the chain contour length. Thus, there is a range of strain amplitudes over which normal force thickening is observed. Lastly, the proposed mechanism for the increased normal forces is the enhancement of bead collisions in an oscillatory flow.

Klein et al. ${ }^{1}$ measured normal force increases during oscillatory shear of a brush-brush system using the SFA. Normal force increases were observed for both compressed and uncompressed brushes. In our simulations we found that the critical parameter governing the brush thickening at large Wi for compressed brushes was the strain amplitude, $\mathrm{Wi} / \omega$ or $\mathrm{A}$. In the experiments of Klein et al. ${ }^{1}$ the strain amplitude is in the range 5-12.6 (or $\omega / \mathrm{Wi}=0.08-0.2$ ). We note that our calculation of the strain amplitude for the system of Klein et al. does not require the use of a relaxation time $\tau_{1}$ since $\mathrm{Wi} / \omega$ is equal to the dimensional shear rate

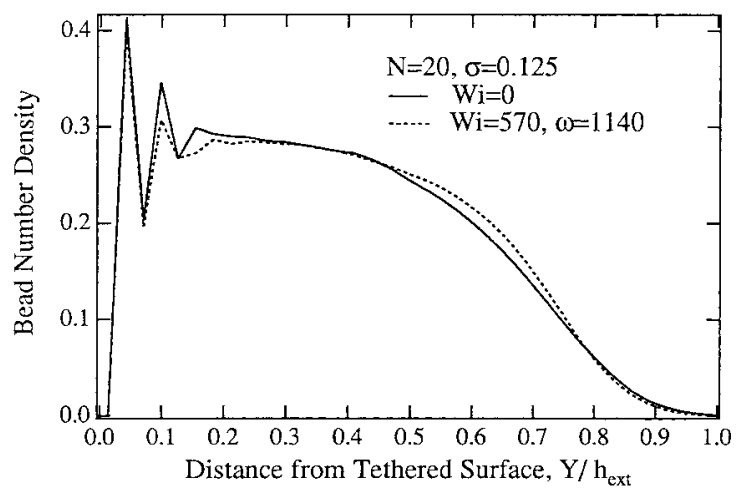

Figure 18. Bead density versus distance from a tethered surface for oscillated uncompressed brushes.

divided by the dimensional frequency. A crude lower bound for $\tau_{1}$ can be constructed by comparing to the experiments of Pelletier et al. ${ }^{8}$ for a similar brush system ${ }^{54}$ which suggested that $\tau_{1}>0.01 \mathrm{~s}$. Using $\tau_{1}=$ $0.01 \mathrm{~s}$ for the Klein et al. ${ }^{1}$ system gives $\mathrm{Wi}=50-100$. We stress that the exact value of the Wi is not necessary to compare to our simulations and scalings; it need be merely $\mathrm{O}(1)$ or larger. If we assume a chain contour length of $140 \mathrm{~nm}$ for the chains in the Klein et al. experiments, our scalings predict normal force increases for strain amplitudes in the range $0.5 \leq \mathrm{A} \leq 2$. There are several reasons why our predicted bound for the largest amplitude is in conflict with the experiments. Foremost is that the oscillatory simulations have very crude hydrodynamics, and the neglect of screening in the brush is a poor assumption at large amplitudes where the chains begin to feel their tether and transmit large stresses to the fluid. Secondly, the scal ing bound for the largest amplitude at which thickening occurs (eq 12) is also an approximation for the simulation data which can be seen in Figure 14. Further SFA experiments which systematically vary the strain amplitude at large Wi would provide a more stringent test of our proposed mechanisms for normal force thickening in oscillatory flow.

3.3.3. Uncompressed Tethered Chains. The shearinduced collision process described for the compressed chains should produce brush thickening in brushes which are not compressed by the upper surface. However, in Figure 14 we see that the range of frequencies, at which a normal stress increase is predicted, will decrease with increasing gap width (in agreement with the scalings presented in the previous section). In Figure 18 we compare the bead density profile of an uncompressed brush at equilibrium and under oscillation at $\omega=1140, \mathrm{Wi}=570(\omega / \mathrm{Wi}=2)$ and $\sigma=0.125$. Under oscillation we observe an increase in the mean thickness of the brush, but the diffuse tail region changes very little. We have observed the same trend for brushes at higher areal fractions. ${ }^{46}$ We found that at higher frequencies the density profile monotonically approaches the equilibrium profile. At lower frequencies the brush thickness decreases. The data in Figure 18 that represent the largest thickening witnessed are very modest.

In the experiments of Klein et al. ${ }^{1}$ they observed increases in the normal force for uncompressed brushes, suggesting a swelling by nearly $25 \%$. The polystyrene chains in the experiments contain approximately 140 Kuhn steps ${ }^{1,46}$ which is larger than the systems we can currently simulate, and so we speculate on the expected 
simulation trends for larger N. Very few collisions occur in the diffuse tail region of the density profile. As $\mathrm{N}$ increases, the tail region decreases (relative to the total profile height ${ }^{55}$ ), and this should give rise to more collisions. Furthermore, the longer chains could be oscillated at lower frequencies before a chain's finite extensibility comes into play. Further simulations are required to confirm these speculations.

\section{Summary}

We have presented simulation and scaling results for a single polymer brush under steady and oscillatory shear. Under steady shear the deformation of the brush proceeded in two steps: chains tilt in the flow direction followed by a physical thinning of the brush and a blunting of the density profiles. Brushes at smaller areal fractions deform at a smaller value of Wi due to the increased penetration of the vel ocity into the brush. Many of these trends had been witnessed in previous simulations, ${ }^{16,31-38}$ but in addition we derived scalings for brush rheology observed in the simulations and compared steady to oscillatory shear. As in previous simulations, ${ }^{16,31-37}$ we found no brush thickening under steady shearing which contrasts with many theor etical studies. ${ }^{12,14,15,17}$ We developed a scaling to explain the initial increase in the viscosity upon compression to near $60 \%$ and decrease occurring thereafter. The viscosity is determined by the characteristic shear rate which is the ratio of the top plate velocity, $\mathrm{U}$, divided by the characteristic distance over which the velocity decays, namely the fluid region above an uncompressed brush and the pore size in a compressed brush. Compressed brushes sheared at small Wi had smaller shear than normal forces, and we developed a scaling which shows an abrupt increase in the shear stress at small gaps. These trends were in qualitative agreement with the experimental results of Klein et al. ${ }^{2}$ which were performed on a brush-brush system under steady shear. Our simulations were performed for a single brush compressed by a smooth wall, and thus the effects of interbrush entanglements were not captured. Our trends for the shear and normal stresses of a compressed brush under steady shear were in qualitative agreement with recent mol ecular dynamics simulations by Grest $^{36,37}$ for a brush-brush system.

We next turned to the dynamics of oscillation to explain the dramatic normal force increases reported by Klein et al. ${ }^{1}$ during oscillatory shear flow of both compressed and uncompressed polymer brushes. Large increases in the normal stress and bead density near the upward surface occurred during oscillatory shearing of compressed brushes at large Wi and frequencies, $\omega$. Shear-induced collisions of beads in the brush were identified as the cause of an increased osmotic pressure in the brush with concomitant swelling. The maximum increase occurs at a critical ratio of $\omega$ to Wi, and thus strain amplitude, $\mathrm{A}=\mathrm{Wi} / \omega$, determined the dynamics during oscillatory flow. Scalings were determined for the range of A over which the simulated normal stresses increased. At large A (small $\omega / \mathrm{Wi}$ ) the onset of the normal stress increases were limited by the chain extensibility. The low A limit of normal stress increases was dictated by the onset of collisions in a system of beads. The range of strain amplitudes (5-12.6) where increased normal stresses were observed by Klein at al. ${ }^{1}$ for compressed brushes was larger than the range predicted from our scaling theory for their system (0.5-
2.). We neglected the stress transmitted by the chains to the fluid in our calculations (because of computational resource limits); this is a poor approximation at large amplitudes. The shear-induced collision mechanism did lead to a thickening of uncompressed brushes, but only in the mean thickness and not the tail region. We speculate that larger chains (which have a smaller tail region in the density profile and are not near full extension at equilibrium in the brush) must be simulated to observe thickening throughout the full extent of an uncompressed brush. Thus, we were unable to reproduce the dramatic normal force increases observed by Klein et al. ${ }^{1}$ for uncompressed brushes under oscillation.

A general shortcoming of the present simulations is the required computational time which limited us to studies of small chains and to implement crude hydrodynamics in our oscillatory simulations. Nevertheless, we were able to provide a fundamental physical understanding of why the dynamics of a polymer brush is drastically different in steady and oscillatory shear flow.

Acknowledgment. This material is based upon work supported by the National Science Foundation under Grant No. DMR-9400354 and support for P.S.D. through a Lieberman Fellowship.

\section{References and Notes}

(1) Klein, J .; Perahia, D.; Warburg, S. Nature 1991, 352, 143.

(2) Klein, J .; Kumacheva, E.; Mahaiu, D.; Perahia, D.; Fetters, L. Nature 1994, 370, 634.

(3) Carlson, A.; Hill, C. G.; Olson, N. Biotechnol. Bioeng. 1987, 29, 590.

(4) Napper, D. Polymeric Stabilization of Coll oidal Dispersions; Academic Press: New York, 1983

(5) Inn, Y.; Wang, S. Phys. Rev. Lett. 1996, 76, 467.

(6) Watanabe, H.; Yao, M.; Osaki, K. submitted to Macromolecules 1997, 30, 5905

(7) Potanin, A.; Russel, W. Phys. Rev. E 1995, 52, 730.

(8) Pelletier, E.; Belder, G.; Hadziioannou, G.; Subbotin, A. J Phys. II France 1997, 7, 271.

(9) Granick, S.; Demirel, A.; Cai, L.; Peanasky, J . I srael J . Chem. 1995, 35, 75.

(10) Cai, L.; Peanasky, J .; Granick, S. Trends Polym. Sci. 1996, $4,47$.

(11) Dhinojwala, A.; Cai, L.; Granick, S. Langmuir 1996, 12, 4537.

(12) Kumaran, V. Macromolecules 1993, 26, 2464.

(13) Rabin, Y.; Alexander, S. Europhys. Lett. 1990, 13, 49.

(14) Barrat, J.-L. Macromolecules 1992, 25, 832.

(15) Harden, J .; Cates, M. Phys. Rev. E 1996, 53, 3782.

(16) Doyle, P.; Shaqfeh, S.; Gast, A. Phys. Rev. Lett. 1997, 78, 1182.

(17) Aubouy, M.; Harden, J .; Cates, M. J . Phys. II France 1996, 6, 969.

(18) Varoqui, R.; Dejardin, P. J . Chem. Phys. 1977, 66, 4395.

(19) Cohen Stuuart, M.; Waajen, F.; Cosgrove, T.; Vincent, B. Crowley, T. Macromolecules 1984, 17, 1825.

(20) Anderson, J .; McKenzie, P.; Webber, R. Langmuir 1991, 7 , 162.

(21) Brinkman, H. Appl. Sci. Res. 1947, A1, 27

(22) Wu, D.; Cates, M. Phys. Rev. Lett. 1993, 20, 4142.

(23) Parnas, R.; Cohen, Y. Rheol. Acta 1994, 33, 485.

(24) Fredrickson, G.; Pincus, P. Langmuir 1991, 7, 786.

(25) Klein, J .; Kamiyama, Y.; Yoshizawa, H.; I sraelachvili, J .; Fredrickson, G.; Pincus, P.; Fetters, L. Macromol ecules 1993, 26, 5552.

(26) Milner, S. Macromolecules 1991, 24, 3704.

(27) Milner, S.; Witten, T.; Cates, M. Macromolecules 1988, 21 , 2610.

(28) Skvortsov, A.; Gorbunov, I.; Pavlushkov, E.; Zuhlina, O.; Borisov, V.; Priamitsyn, V. Vysokomol. Soedon. Ser. A 1988, 30, 1615.

(29) Alexander, S. J . Phys. (Paris) 1977, 38, 977.

(30) de Gennes, P. Macromolecules 1980, 13, 1069

(31) Lai, P.-Y.; Binder, K. J . Chem. Phys. 1993, 98, 2366.

(32) Lai, P.-Y.; Lai, C.-Y. Phys. Rev. E 1996, 54, 6958. 
5486 Doyle et al.

(33) Miao, L.; Guo, H.; Zuckerman, M. Macromol ecules 1996, 29, 2289.

(34) Peters, G.; Tildesley, D. Phys. Rev. E 1995, 52, 1882.

(35) Peters, G.; Tildesley, D. Phys. Rev. E 1996, 54, 5493.

(36) Grest, G. Phys. Rev. Lett. 1996, 76, 4979.

(37) Grest, G. MRS Proceedings 1997, 464 (Polymer Brushes in Strong Shear Flow), p 71.

(38) Neelov, I.; Borisov, O.; Binder, K. 1997, preprint.

(39) Doyle, P.; Shaqfeh, E.; Gast, A. J . Fluid Mech. 1997, 334 251.

(40) Gardiner, C. W. Handbook of Stochastic Methods, 2nd ed.; Springer-Verlag: New York, 1985.

(41) Russel, W.; Saville, D.; Schowalter, W. Colloidal Dispersions; Cambridge University Press: Cambridge, 1989.

(42) Liu, T. J . Chem. Phys. 1989, 90, 5826.

(43) Weeks, J .; Chandler, D.; Andersen, H.J . Chem. Phys. 1971, $54,5237$.

(44) Allen, M. P.; Tildesley, D.J . Computer Simulation of Liquids, Clarendon Press: Oxford, 1987.
Macromolecules, Vol. 31, No. 16, 1998

(45) Koch, D.; Brady, J . AIChE J . 1986, 32, 575.

(46) Doyle, P. S. Ph.D. Thesis, Stanford U niversity, Stanford, CA 1997.

(47) Murat, M.; Grest, G. Phys. Rev. Lett. 1989, 63, 1074

(48) Grest, G.; Murat, M. In: Monte Carlo and Molecular Dynamics Simulations in Polymer Science; Binder, K., Ed.; Oxford University: Oxford, 1995

(49) Milner, S. Europhys. Lett. 1988, 7, 695.

(50) Milner, S. Science 1991, 22, 905.

(51) Alexander, S. J . Phys. (Paris) 1977, 38, 983.

(52) Leighton, D.; Acrivos, A. J . Fluid Mech. 1987, 177, 109.

(53) Leighton, D.; Acrivos, A. J . Fluid Mech. 1987, 177, 415.

(54) The chains in brushes of Pelletier et al. ${ }^{8}$ are approximately half the length of the chains in Klein et al. ${ }^{1}$ and at comparable grafting densities. The lower bound for $\tau_{1}$ is deduced from the trends in the loss and storage modulus in Pelletier et al. ${ }^{8}$

(55) Milner, S. J . Chem. Soc. Faraday Trans. 1990, 86, 1349.

MA970821X 